\title{
Optimal reconstruction of concentrations, gradients and reaction rates from particle distributions
}

\author{
D. Fernàndez-Garcia *, X. Sanchez-Vila \\ Department of Geotechnical Engineering and Geosciences, Technical University of Catalonia, Gran Capità s/n, 08034 Barcelona, Spain
}

\section{A R T I C L E I N F O}

\section{Article history:}

Received 14 December 2009

Received in revised form 20 April 2010

Accepted 5 May 2010

Available online 19 May 2010

\section{Keywords:}

Particle tracking

Reactive transport

Mixing

Heterogeneity

Subsurface hydrology

\begin{abstract}
A B S T R A C T
Random walk particle tracking methodologies to simulate solute transport of conservative species constitute an attractive alternative for their computational efficiency and absence of numerical dispersion. Yet, problems stemming from the reconstruction of concentrations from particle distributions have typically prevented its use in reactive transport problems. The numerical problem mainly arises from the need to first reconstruct the concentrations of species/components from a discrete number of particles, which is an error prone process, and then computing a spatial functional of the concentrations and/or its derivatives (either spatial or temporal). Errors are then propagated, so that common strategies to reconstruct this functional require an unfeasible amount of particles when dealing with nonlinear reactive transport problems. In this context, this article presents a methodology to directly reconstruct this functional based on kernel density estimators. The methodology mitigates the error propagation in the evaluation of the functional by avoiding the prior estimation of the actual concentrations of species. The multivariate kernel associated with the corresponding functional depends on the size of the support volume, which defines the area over which a given particle can influence the functional. The shape of the kernel functions and the size of the support volume determines the degree of smoothing, which is optimized to obtain the best unbiased predictor of the functional using an iterative plug-in support volume selector. We applied the methodology to directly reconstruct the reaction rates of a precipitation/dissolution problem involving the mixing of two different waters carrying two aqueous species in chemical equilibrium and moving through a randomly heterogeneous porous medium.
\end{abstract}

(c) 2010 Elsevier B.V. All rights reserved.

\section{Introduction}

Solute transport of conservative solutes has been traditionally studied using the Advection-Dispersion Equation (ADE) which is derived based on local arguments. In the last decades, though, this equation has been greatly questioned (e.g., Neuman and Tartakovsky, 2008, and references therein) and a number of non-local formulations have appeared as alternatives to describe effective transport of conservative

\footnotetext{
* Corresponding author.

E-mail addresses: daniel.fernandez.g@upc.edu (D. Fernàndez-Garcia), xavier.sanchez-vila@upc.edu (X. Sanchez-Vila).
}

species at intermediate distances. These formulations include Continuous Time Random Walks (CTRW) (Berkowitz et al., 2006), Fractional Advection-Dispersion Equations (fADE) (Benson et al., 2000), Multi-Rate Mass Transfer models (MRMT) (Haggerty and Gorelick, 1995) and memory functions (Carrera et al., 1998).

Irrespectively of the choice of the underlying Partial Differential Equation governing the problem, transport equations are solved by means of numerical methods. In this context, Random Walk Particle Tracking Methodologies (RWPT) constitute an attractive technique for their computational efficiency and absence of numerical dispersion. These methods simulate solute transport by tracking in time a large number of particles injected into the system, each one with a predefined associated mass. At any time step (with $\Delta t$ being 
either constant or random), the particle moves due to the sum of two terms, one being deterministic (loosely associated to advective processes) and the other random (loosely associated to dispersive processes). For any given time, resident concentrations can be recovered by defining a grid and counting the mass per unit volume of the particles that are located within a given support volume. The concentration estimate of a given support is a random variable but eventually converges to the "true value" when the number of particles tends to infinity. In other words, the mean estimation error of this estimator of concentrations decreases with the number of particles. Despite these problems, particle tracking algorithms are a good alternative compared to other numerical methods such as finite elements or finite differences (e.g., Salamon et al., 2006a).

Particle tracking algorithms are a convenient alternative to study molecular diffusion problems. In such cases particles can be associated to molecules. This would mean that a mole of solute should be discretized into $N_{A}$ (Avogrado's number, $N_{A}=6.0210^{23}$ ) molecules. In all practical applications found in the literature the number of particles used to discretized a given mass is more in the order of $10^{4}$ to $10^{7}$ (e.g., Riva et al., 2008). The traditional approach is based on considering that each particle actually represents a group of molecules. In this case particles have a representative size. This concept has been included into a family of methods named smoothed-particle

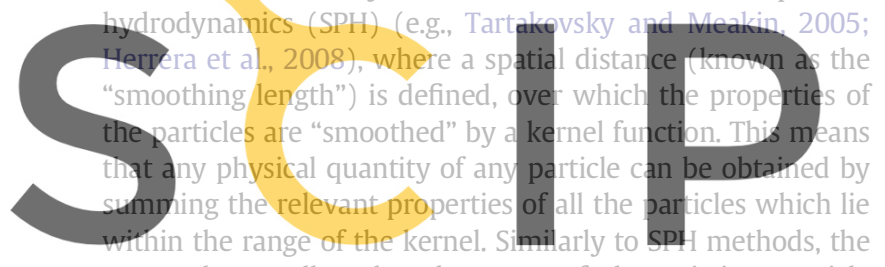
approach actually taken by most of the existing particle Register for fres (eqt https / www.scipedial.com to share in common that they provide noisy estimates of the spatial or temporal distribution of concentrations. This is particularly disturbing when the objective is not actually estimating concentrations, but rather spatial or temporal derivatives, which is the case in most applications regarding stochastic hydrology or reactive transport.

The alternative we propose is based on considering that the particles being tracked are just a subsample of the population. This renders the problem of going from particles to concentrations to be just a reconstruction problem, where the spatial or temporal distribution of a given variable must be inferred from the observation of the spatial or temporal location of a relatively small subsample. The initial selection of the particles to be tracked (amongst all the possible ones) becomes an uncorrelated random sampling process, each particle/molecule being given the same probability. This way, the larger the concentration in a given volume, the larger the number of particles finally selected (assuming a sufficient number of particles is considered). Since dispersion processes are intrinsically random, a different realization of the solute transport problem can just be seen as coming from a different sample extraction from the initial population. The reconstruction problem considered only accounts for subsampling and therefore disregards the accuracy issues related to the flow problem solution (discretization errors and convergence).
The methods to carry out such reconstruction can be borrowed from many other problems in science. A widely used family of methods is based on kernel particle filters, where kernel density estimators (KDE) can be used to reconstruct the posterior PDF's of the variable of interest. Fields of application are signal processing, wireless communication, and robotics among others (see e.g., Chang and Ansari, 2005; Stoessel and Sagerer, 2006) and it is the approach taken in this paper. Particle filters can be considered a generalization of the traditional Kalman filtering methods. A compilation of different particle filtering methods with a comparison with Kalman filters and other types of gridfiltering methods can be found in a lucid discussion by Arulampalam et al. (2002).

In a KDE based approach, the multivariate kernel associated with the corresponding functional (e.g., the pdf of concentration estimator) depends on the size of the support volume, which defines the area over which a given particle can influence the functional. The shape of the kernel functions and the size of the support volume determines the degree of smoothing which is optimized to obtain the best unbiased predictor of the functional using an iterative plug-in support volume selector. Arguably, the main advantage of Kernel Density filters is its ability to directly reconstruct optimally not only concentrations, but also spatial and temporal derivatives by optimizing the kernel functions in

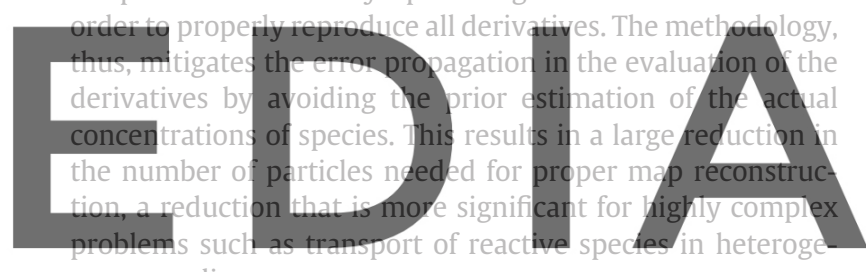

neous media.

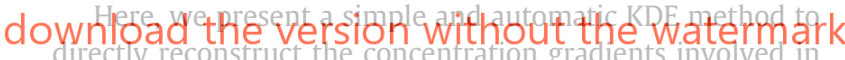

transport quantities such as memory functions, mixing indexes and reaction rates, based on the estimation of marginal and conditional density functions of the concentration derivatives. The paper is structured as follows. First, Section 2 describes the target transport quantities to be estimated. Section 3 presents the theoretical framework and development of the KDE methodology. Finally, Section 4 shows an application of the method conducive to reconstruct the reaction rates of a precipitation/dissolution problem involving the mixing of two different waters in chemical equilibrium and moving through a homogeneous and a randomly heterogeneous porous medium. The homogeneous media is used to contrast the method against traditional approaches and analytical solutions. The heterogeneous media is used to illustrate the effect of mixing on reactive transport in a real field setting.

\section{Background and motivation}

There are a number of key hydrogeological problems where we are actually interested in directly obtaining a good and efficient estimator of the derivatives or gradients. In the last few years a great interest has arisen in the literature concerning memory functions. This concept is directly related to the slope of the breakthrough curve (see e.g. 
Haggerty et al., 2000; Dentz and Berkowitz, 2003), s which can be written as

$s=\frac{d \log c}{d \log t}=\frac{t}{c} \frac{d c}{d t}$

Spatial derivatives of concentration are key when the concept of dilution is introduced into the solute transport picture. Kitanidis (1994) defined the dilution index E, a measure of the degree of dilution in the system whose derivative is given in terms of the integral upon the full domain $\Omega$ of a combination of the spatial derivatives of concentrations through

$\frac{d \ln E}{d t}=\int_{\Omega} \frac{1}{c} \nabla c^{t} \mathbf{D} \nabla c d \mathbf{x}$

The concept of dilution is directly related to that of concentration variance, $\sigma_{r}^{2}$, defined by Kapoor and Gelhar (1994), Kapoor and Kitanidis (1998). Remarkably, $\sigma_{c}^{2}$ follows an ADE type with a source term (or destruction term), $f_{\sigma_{c}^{2}}$ given by

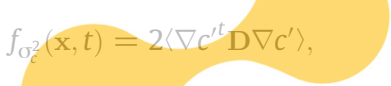

where $\langle\cdot\rangle$ denotes the expectation operator, and $c^{\prime}$ is the deviation of the concentration field with respect to the mean field, obtained by solving a macrodispersive ADE. Dilution is also the cause that the polf (the piobs of concentrations displays a betaFiorotto and Caroni, 2002; Bellin the $p d f$ of concentrations in het defined as the ensemble mean ez-Vila et al., 2009) which it

equation with a source term $f_{p d f}$ given as

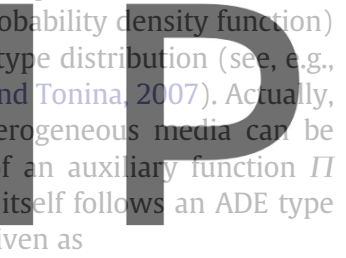
Register for freer att https//WwW.scipedia.com $f_{(4)}$ to

Particle tracking approaches can also be applied to reactive transport problems. A particular case when reactive transport can be efficiently handled using particle tracking is the case when transport can be fully defined in terms of conservative quantities called components (see Fernàndez-Garcia et al., 2008, for an example). This is the case for example when all solutes are subject to the same velocity and dispersivity (at any given point) and reactions are instantaneous. In this case, and assuming that the vector of conservative quantities $\mathbf{u}$ follows an ADE, De Simoni et al. (2005) show that the vector of reaction rates per unit volume of fluid, $\mathbf{r}$, can be computed as

$\mathbf{r}(\mathbf{x}, t)=\mathbf{H} \nabla u^{t} \mathbf{D} \nabla u$

$\mathbf{H}$ being the Hessian matrix involving the second derivatives of concentration of solutes with respect to concentrations of components. This matrix is obtained from a chemical speciation process. Eq. (5) was further extended by Donado et al. (2009) to obtain reaction rates when the governing equation for the conservative quantities is a MRMT model. We note that there are other alternative approaches to model reactive transport with particle tracking avoiding the prior calculation of concentrations (e.g. Gillespie, 1977). Still, concentrations of reactants and products should be computed optimally afterwards.

From the non-exhaustive list presented it is clear that a number of problems in solute transport involve the computation of concentration gradients. Thus, it is crucial to find an optimal way to estimate these derivatives/gradients whenever a particle tracking approach is used to solve the underlying governing transport equation. The method we propose is capable of finding optimal estimations of the concentration gradients. It is true that some of the problems addressed involve the computation of products of derivatives. While it is true that the optimal of the product is not the product of optimals, we believe that our approach can be used even for these problems to provide suboptimal estimates of quantities such as Eqs. (1), (2) and (5).

\section{Kernel density estimators}

Particle tracking techniques produce discrete distributions of particle attributes (mass) that have to be converted to a continuous distribution of concentrations. The mathematical representation of the concentration field from particle distributions depends on the type of observation. Particle clouds observed at given times $t_{0}$ yield resident concentrations $c_{r}$ (concentrations averaged over a support volume), whereas particles passing through control surfaces located at

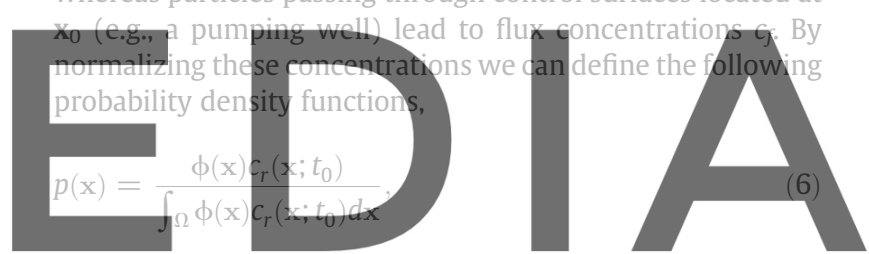

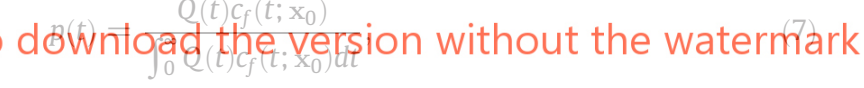

where $p(\mathbf{x})$ is the probability of finding a solute mass within the support volume $[\mathbf{x}, \mathbf{x}+\mathrm{d} \mathbf{x}]$ at a given time $t$, and $p(t)$ is the probability of finding a solute mass within the time interval $[t, t+\mathrm{d} t]$ at a given control location. $\phi(\mathbf{x})$ is the porosity and $Q(t)$ is the flow rate at the outlet location. The particle mass is then related to the normalized concentrations by

$$
\begin{aligned}
& p(\mathbf{x})=\frac{m_{p}\left(\mathbf{x}_{p}\right)}{M_{t}} E\left\{\delta\left(\mathbf{x}-\mathbf{X}_{p}\right)\right\}, \\
& p(t)=\frac{m_{p}\left(T_{p}\right)}{M_{a}} E\left\{\delta\left(t-T_{p}\right)\right\},
\end{aligned}
$$

where $E\{\cdot\}$ is the expectation operator over many injected random particles, $\mathbf{X}_{p}$ is the $p$ th-particle location at time $t, T_{p}$ is the first passage time of the $p$ th-particle crossing the control surface, $M_{t}$ is the total mass in the domain $\Omega$ at time $t$, and $M_{a}$ is the total mass passing through the control surface,

$$
\begin{aligned}
& M_{t}=\int_{\Omega} \phi(\mathbf{x}) c_{r}\left(\mathrm{x} ; t_{0}\right) d \mathbf{x} \\
& M_{a}=\int_{0}^{\infty} Q(t) c_{f}\left(t ; \mathrm{x}_{0}\right) d t
\end{aligned}
$$


A natural estimate of the probability density functions $p(t)$ or $p(\mathbf{x})$ is the relative frequency of mass, which basically consists in counting the particle mass falling into a given support. In the traditional approach to recover concentrations from particle distributions, this support is defined based on a given discretization of the domain in space and/or time so that $p(t)$ and $p(\mathbf{x})$ are only evaluated at the centroid of the discretization elements. This is mathematically written as

$p\left(\mathbf{x}_{j}\right) \approx \hat{p}\left(\mathbf{x}_{j}\right) \equiv \frac{1}{M_{t}} \sum_{p} \frac{m_{p} I\left\{\mathbf{x}_{p} \in B_{j}\right\}}{\Delta V_{j}}$

$p\left(t_{j}\right) \approx \hat{p}\left(t_{j}\right) \equiv \frac{1}{M_{a}} \sum_{p} \frac{m_{p} I\left\{T_{p} \in B_{j}\right\}}{\Delta t_{j}}$,

where the overhat indicates the estimator, $B_{j}$ is respectively the support volume or the time interval, $I\{\cdot\}$ is an indicator function defined as

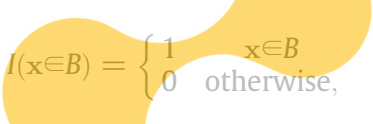

and $\mathbf{x}_{j}$ and $t_{j}$ denote the centroid of the $j$ th discretization element in space and time with sizes $\Delta V_{j}$ and $\Delta t_{j}$ respectively. In the limit, for infinitesimal supports, $I / \Delta V_{j}$ and $I / \Delta t_{j}$ approach a $\delta$-function estimators depend on the don mass of particles falling into the support combined with a finite

very noisy estimates, whereas an increase in th tends to oversmoth (over or underestimate) the estimated

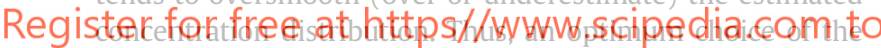
support size exists. In this context, kernel density estimators (KDE) provide a convenient mathematical framework to obtain this optimal support.

The KDE approach starts by generalizing the previous estimators as

$p(\mathbf{x}) \approx \hat{p}(\mathbf{x}) \equiv \frac{1}{M_{t}} \sum_{p} m_{p} K_{H}\left(\mathbf{x}-\mathbf{X}_{p}\right)$

$p(t) \approx \hat{p}(t) \equiv \frac{1}{M_{a}} \sum_{p} m_{p} K_{H}\left(t-T_{p}\right)$,

where $K_{H}\left(\mathbf{x}-\mathbf{X}_{p}\right)$ and $K_{H}\left(t-T_{p}\right)$ are kernels or weighting functions dependent on the separation distance between the particle position/time and the point of estimation (e.g., Hardle, 1990). The traditional approach is recovered by using $K_{H}=I\{\cdot\} /$ $\Delta V$, which is known as the box kernel function. The difference between the formulation given by Eqs. (12)-(13) and Eqs. (15)-(16) is that the averaging of the kernels can be done also at a point different from the discretization element centroid. Thus, albeit the traditional box model introduces discontinuities at the box edges, kernel estimators produces smooth functions of space and/or time. These kernel functions are weighting functions of the particle mass that defines its region of influence. They are usually defined to be symmetric density functions whose shape and size is parametrized based on a smoothing parameter, $H$. Thus,

$\int K_{H}(\mathbf{s}) d \mathbf{s}=1$

where the integral extends over the full domain. This parameter $H$ defines how the particle attributes can influence the concentrations. For flux concentrations, $K_{H}$ is a univariate distribution of particle arrival times and therefore $H$ is a scalar parameter. For resident concentrations, $\mathbf{H}$ is a symmetric positive definite $d \times d$ matrix and $K_{H}$ is a $d$-variate distribution of the particle position in space ( $d$ being the space dimension). The Kernel function can generally be expressed by means of elementary Kernel functions, $K$, defined as

$K_{H}(\mathbf{s})=\mathbf{H}^{-1 / 2} K(\mathbf{V} \mathbf{s})$,

$\mathbf{V} \cdot \mathbf{V}^{t}=\mathbf{H}$.

The shape of the elementary Kernel functions, $K$ and the choice of $\mathbf{H}$ determines the degree of smoothing of concentrations. A variety of Kernel functions can be used to generate smooth concentrations (e.g., Hardle, 1990). Well-known models are the Triangle model and the Gaussian model, respectively written as

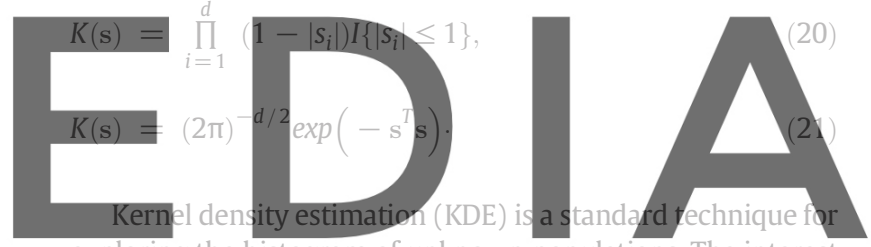

exploring the histogram of unknown populations. The interest of KDE methods to particle tracking methodologies is manydownloasch thes versionewithouts the waterimark multimodal distributions (e.g., the presence of double peaks) as well as the identification of non-Fickian features of solute transport (e.g., pronounced tailing in breakthrough curves); (2) It directly provides not only good estimates of concentrations but also of their functionals (e.g., derivatives, gradients, dilution and corresponding indexes); and (3) It can be used to directly select the optimal degree of smoothing of the concentration and/or their derivatives based on data in an automatic way. The latter is briefly described in this section.

\subsection{Optimal estimates of flux concentrations}

Optimal estimates of $p(t)$ can be determined based on an appropriate selection of the smoothing parameter $H$, which is now a scalar parameter because the problem is one-dimensional. This is obtained by minimizing some error measure. A common choice is the Mean Integrated Square Error (MISE), defined as

$\operatorname{MISE}(H)=E\left\{\int(\hat{p}(t)-p(t))^{2} d t\right\}$.

Hereinafter, the limits of integration have been deliberately excluded in all integrals to denote that the domain where optimization is performed is a modeler's choice. As an example, one can be interested in a concentration value that tends asymptotically to a baseline value. Integrating up to infinity 
leads to an unbounded integral. Thus, a cut-off must be imposed. Also, it would be possible to obtain different optimal estimates in prespecified subdomains. In the simulations presented in this paper we have used the full domain.

Since it is known that the choice of $K$ has little effect on the behavior of $\hat{p}$, in Eq. (22) we avoided to write explicitly the dependence of the shape of $K$ in MISE. The reason for this is that kernel functions can be rescaled such that the difference between two KDE estimates obtained using two different kernels is almost negligible (Marron and Nolan, 1989). MISE can be written as the sum of two terms, the integral of variance and the integrated squared bias,

$\operatorname{MISE}(H)=\int \operatorname{Var}[\hat{p}(t)] d t+\int \operatorname{Bias}^{2}[\hat{p}(t)] d t$.

Assuming that all particles carry the same mass $m_{p}$, so that the total mass is $M=N m_{p}$, using a change of variable along with the Taylor expansion of $p(t)$ around $t$, and taking the limit of MISE as $N_{p} H \rightarrow \infty$ and $H \rightarrow 0$, the following asymptotic expressions can be found (e.g., Silverman, 1986; Hardle, 1990), $\operatorname{MISE}(H)=\operatorname{AMISE}(H)+O\left(H^{4}+\left(N_{p} H\right)^{-1}\right)$, (24)

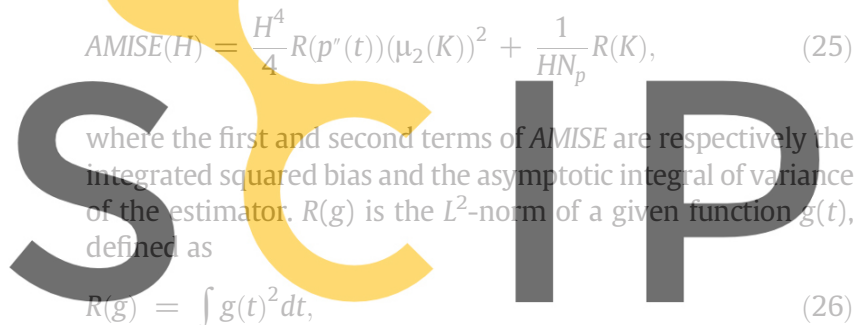

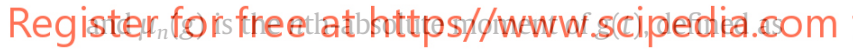

$\mu_{n}(g)=\int t^{n} g(t) d t$.

Hence, the minimization of MISE with respect to $H$ calls for a compromise between oversmoothing (taking a large $H$ to reduce the variance) and undersmoothing (taking a small $H$ to reduce the bias). The optimal choice of the bandwidth, $H^{\text {opt }}$, is then obtained by disregarding higher order terms and minimizing the asymptotic expression of $\operatorname{MISE}(H)$. Setting $\mathrm{d} A M I S E / \mathrm{d} H=0$ yields (Park and Marron, 1990)

$H^{\mathrm{opt}}=\left(\frac{R(K)}{R\left(p^{\prime \prime}\right)\left(\mu_{2}(K)\right)^{2} N_{p}}\right)^{1 / 5}$.

Thus, it turns out that the optimal bandwidth $H^{\text {opt }}$ is inversely proportional to the number of particles used to the power of 0.2 and depends on the unknown function $R\left(p^{\prime \prime}\right)$. In the limit, when $N_{p} \rightarrow \infty$ the optimal bandwidth consistently approaches to zero. $R\left(p^{\prime \prime}\right)$ needs to be further estimated. Several methods can be used for this matter: (1) rule-of-thumb methods (i.e., a guess of the reference distribution $p$ ); (2) cross-validation methods; and (3) plug-in methods. A review of these methods can be found in several papers (e.g., (Jones et al., 1996; Park and Marron, 1990)) and books (e.g., (Hardle, 1990)).

\subsection{Optimal estimates of resident concentrations}

Likewise for flux concentrations, optimal estimates of resident concentrations at time $t, p(\mathbf{x})$, can also be determined based on an appropriate selection of the support volume $\mathbf{H}$, defined based upon an integrated square error measure as

$\operatorname{MISE}(\mathbf{H})=E\left\{\int(\hat{p}(\mathbf{x})-p(\mathbf{x}))^{2} d \mathbf{x}\right\}$.

In a one-dimensional problem, the estimation of resident concentrations is exactly the same as for flux concentrations exchanging $t$ by $x$. Nonetheless, in practice, the spatial distribution of resident concentrations is evaluated in two or three dimensions. In this case, albeit the choice of the smoothing parameter is rather simple for univariate problems, it becomes difficult for multivariate distributions, where it is required to estimate several free parameters. Wand and Jones (1993) found that the choice of $\mathbf{H}$ in twodimensional problems should, in general, account for the curvature and orientation of the true distribution. Moreover, its principal directions could not be chosen effectively using the sample covariance matrix.

Several approaches can be considered to estimate multivariate probability density functions (Wand and Jones, 1994; Sain, 2002; Duong and Hazelton, 2003; Duong et al., 2008). A simple and effective alternative to avoid the difficulties

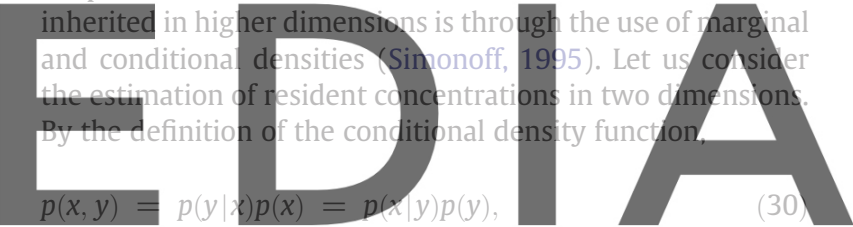

where $p(x \mid y)$ and $p(y \mid x)$ are the conditional density functions, dawn read the versionainithods the watermark

Eq. (30), the bivariate density function can be estimated as (Simonoff, 1995)

$p(x, y)=[p(y \mid x) p(x) p(x \mid y) p(y)]^{1 / 2}$.

The marginal and conditional probability distributions are estimated from the following expressions

$p(x) \approx \hat{p}(x) \equiv \frac{1}{M_{t}} \sum_{p} m_{p} K_{H}\left(x-X_{p}\right)$

$p(y) \approx \hat{p}(y) \equiv \frac{1}{M_{t}} \sum_{p} m_{p} K_{H}\left(y-Y_{p}\right)$

$p(x \mid y) \approx \hat{p}(x \mid y) \equiv \frac{1}{M_{t}(y)} \sum_{p} m_{p} K_{H}\left(x-X_{p}\right)$,

$p(y \mid x) \approx \hat{p}(y \mid x) \equiv \frac{1}{M_{t}(x)} \sum_{p} m_{p} K_{H}\left(y-Y_{p}\right)$

$M_{t}(x)=\int \phi c(x, y) d y$

$M_{t}(y)=\int \phi c(x, y) d x$

$M_{t}=\iint \phi c(x, y) d x d y$ 
where $K_{H}$ is the kernel for univariate distributions $(d=1)$. From this, we see that the problem of estimating multivariate distributions can be easily reduced to the simple estimation of the corresponding marginal and conditional univariate distributions. We refer to Simonoff (1995) for the details on the numerical implementation of Eq. (31).

\subsection{Optimal estimates of the derivatives of flux concentrations}

By knowing $p(t)$ and its derivative with respect to time we can determine the time derivative of the flux concentrations, i.e., the slope of a breakthrough curve, through

$\frac{d c_{f}\left(t ; \mathbf{x}_{0}\right)}{d t}=\frac{M_{a}}{Q(t)}\left(\frac{d p(t)}{d t}-p(t) \frac{d \ln Q(t)}{d t}\right)$,

which is obtained by taking the derivative of $\mathrm{Eq}$. (7). In this context, KDE methods are capable to provide a direct, data-based calculation of the optimal of the derivatives of $p(t)$ without having to use the estimates of concentrations $\hat{p}(t)$. The kernel density estimate $\hat{p}^{(k)}(t)$ of the $k$ th derivative of a univariate probability density function $p(t)$ is (e.g., Engel et al., 1994)

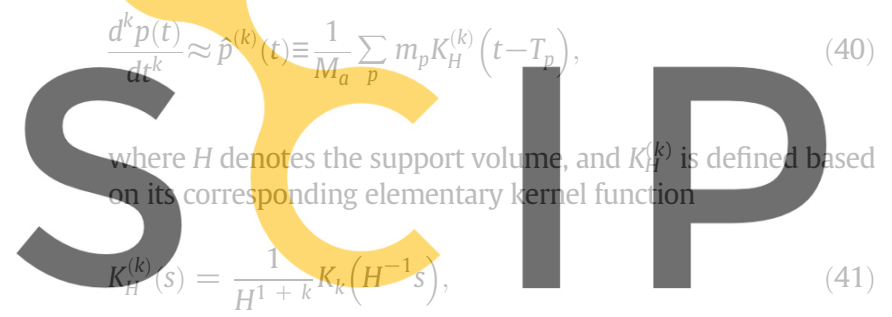

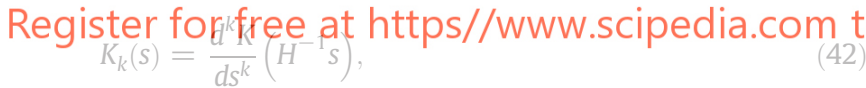

that satisfies

$$
\int K_{k}(s) s^{j} d s= \begin{cases}0 & \text { for } j=0, \ldots, k-1, k+1, \ldots, m-1 \\ (-1)^{k} k ! & \text { for } j=k \\ \mu_{m}\left(K_{k}\right) \neq 0 & \text { for } j=m .\end{cases}
$$

Eq. (42) establishes the relationship between the elementary kernel function of $\hat{p}^{(k)}(t)$ and that of $\hat{p}(t)$, and requires $K$ to be $k$ th differentiable. Using again the mean integrated squared error criterion to evaluate the expected error of the estimator, and assuming that all particles carry the same mass, the following optimal support can be derived (Engel et al., 1994),

$H^{\mathrm{opt}}=\left(\frac{(2 k+1) R\left(K_{k}\right) m !^{2}}{2(m-k)\left(\mu_{m}\left(K_{k}\right)\right)^{2} R\left(p^{(m)}\right) N_{p}}\right)^{1 /(2 m+1)}$.

For completeness, the derivation of Eq. (44) is also given in Appendix A. The important point here is to note that the optimal support associated with the derivative of the density function $\hat{p}^{(k)}$ is not the same as for the density function $\hat{p}$. The fluctuations associated with the derivative of a density function are larger than those of the density function and, therefore, its corresponding optimal support should be increased to provide the same degree of smoothing.

\subsection{Optimal estimates of the gradients of resident concentrations}

Similar to the calculation of flux concentrations, by using Eq. (6), the gradients of the resident concentrations can be written as a function of $p(\mathbf{x})$,

$\nabla c_{r}(\mathbf{x} ; t)=\frac{M_{t}}{\phi(\mathbf{x})}(\nabla p(\mathbf{x})-p(\mathbf{x}) \nabla \ln \phi(\mathbf{x}))$.

Here, $\nabla p(\mathbf{x})$ is a function of space and therefore its estimation is difficult when the problem is not onedimensional. From Eq. (30), and after some algebra,

$\partial_{x} p(x, y)=\operatorname{sgn}\left(\frac{d p(x \mid y)}{d x}\right)\left[p(y \mid x) \frac{d p(x)}{d x} \frac{d p(x \mid y)}{d x} p(y)\right]^{1 / 2}$

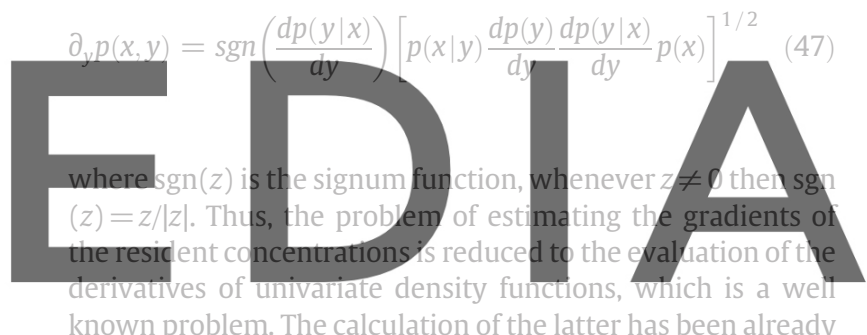

dowastiordintipeiversiond withodit the watermark

\section{Computational investigations}

Particle tracking simulations of a fairly complex multispecies reactive system were conducted in a synthetic aquifer to illustrate the process of reconstructing the reaction rates of chemical species from particle mass distributions. The discussion is organized as follows: Firstly, the reactive transport problem is described in Section 4.1. Then, we examine the performance of the proposed KDE method by contrasting particle tracking solutions of the reaction rates taking place in a homogeneous medium against its corresponding analytical solution (Section 4.2). Finally, in Section 4.3, we explore the effects of heterogeneity and the choice of the transport model on the behavior of the reactive system.

\subsection{Reactive transport problem}

We consider a dissolution/precipitation problem involving the mixing of two different waters. Each water carries in solution two aqueous species, $B_{1}$ and $B_{2}$, in instantaneous local equilibrium with a solid mineral $M_{3}$. The corresponding reaction is

$v_{1} B_{1}+v_{2} B_{2} \rightleftarrows M_{3}$. 
Without loss of generality we consider $\nu_{1}=\nu_{2}=1$. The law of mass action implies that the activities of both aqueous species, $\left\{B_{i}\right\}(i=1,2)$, must satisfy the following condition

$K_{e q}=\left\{B_{1}\right\}\left\{B_{2}\right\}$

where $K_{e q}$ is the equilibrium constant. Assuming that the solution is diluted, then we can assume unit activity coefficients and reformulate Eq. (48) in terms of concentrations

$K_{e q}=c_{1} c_{2}$

In this particular transport problem, the mixing of any two waters in equilibrium with the mineral leads to oversaturation of the resulting mixture. Precipitation then takes place instantaneously in order to re-equilibrate the system.

The migration of the two aqueous species is described by means of a mass transfer model. Mass transfer models are capable to simulate a large variety of processes, including composite diffusive processes (Haggerty and Gorelick, 1995), slow advection (Willmann et al., 2008), and subgrid model heterogeneity (Fernàndez-Garcia et al., 2009). Here, we chose the widely used single-rate mass transfer model. This model considers an overlapped continuum media formed by a mobile domain, where advection-dispersion takes place, and one immobile domain, where mass can be transferred to and temporarily be trapped. Thus, the partial differential
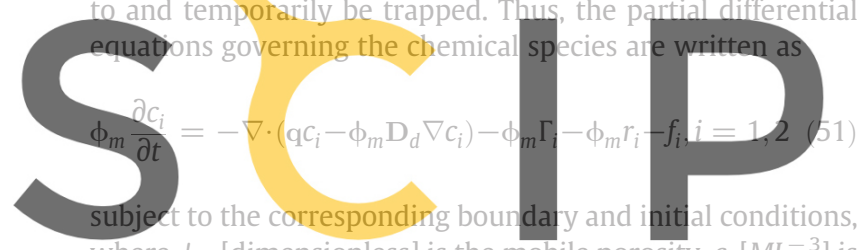

where $\phi_{m}$ [dimensionless] is the mobile porosity, $c_{i}\left[M L^{-3}\right]$ is the volume-averaged concentration of the $i$ th reactive specie,

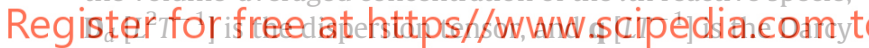
velocity. The functions $r_{i}, f_{i}$, and $\Gamma_{i}\left[M L^{-3} T^{-1}\right]$ are source/sink terms accounting for the mass removal due to chemical reactions, extractions, and mass transfer processes. The source/ sink term $\Gamma_{i}$ is

$\Gamma_{i}(\mathbf{x}, t)=\beta \frac{\partial c_{i m, i}}{\partial t}, i=1,2$,

which describes the mass exchange between the mobile and the immobile domain of the $i$ th specie (per unit of aquifer volume), $\beta$ [dimensionless] is the field capacity of the immobile domain, and $c_{i m, i}\left[M L^{-3}\right]$ is the immobile resident concentration of the $i$ th specie. The mass transfer equation needed to close Eq. (51) together with Eq. (52) is written as

$\frac{\partial c_{i m, i}}{\partial t}=\alpha\left[c_{i}-c_{i m, i}\right]-r_{i m, i}, i=1,2$,

where the reaction rate $r_{i m, i}$ is the source/sink term $\left[M L^{-3} T^{-1}\right]$ that accounts for the solute removed from the immobile domain by precipitation (i.e., precipitated mass per unit of fluid volume and time), and $\alpha\left[T^{-1}\right]$ is the first-order mass transfer coefficient.

We assume that both species sample the same advective process (a counterexample would be colloidal material or sorptive solutes) and both have the same dispersion coefficient. Then, following (De Simoni et al., 2005) and Willmann et al. (2009), this system can be fully defined by means of conservative concentration components, defined as

$u=c_{1}-c_{2}, u_{i m}=c_{i m, 1}-c_{i m, 2}$.

Subtracting the two mass balance equations governing $c_{1}$ and $c_{2}$, the conservative components $u$ and $u_{i m}$ follow conservative equations, i.e., without the presence of $r_{i}$ and $f_{i}$ terms in Eq. (53). Once $u$ and $u_{i m}$ are obtained from solving the conservative transport problem subject to initial and boundary conditions, the species concentrations in the mobile and immobile domains can be explicitly computed by speciation as

$c_{i}=(-1)^{i-1} \frac{u}{2}+\frac{1}{2}\left(u^{2}+4 K_{e q}\right)^{1 / 2}, i=1,2$,
$c_{i m, i}=(-1)^{i-1} \frac{u_{i m}}{2}+\frac{1}{2}\left(\left(u_{i m}\right)^{2}+4 K_{e q}\right)^{1 / 2}, i=1,2$.

Applying the chain rule to Eq. (53) and assuming that $K_{e q}$ is uniform in space and time, Willmann et al. (2009) found the following expression of the reaction rates taking place at the mobile and immobile domains,

$r_{m}(u)=f_{\text {chm }}(u) f_{\text {mix }}(u)$ (57)
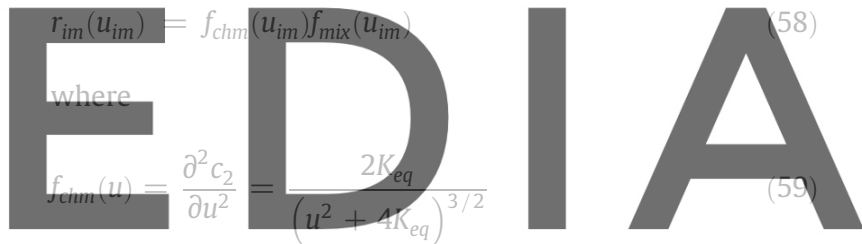

\section{dowithoad the ${ }^{2}$ version without the watermpark}

In Eq. (58), $f_{\text {mix }}(\cdot)$ term is a measure of mixing, while $f_{\text {chm }}(\cdot)$ term is directiy associated with the chemistry of the system and has an explicit expression in terms of the conservative concentration components. Total reaction rates can then be computed as

$r=\frac{\phi_{m}}{\phi_{t o t}} r_{m}+\frac{\phi_{i m}}{\phi_{t o t}} r_{i m}$

where $r$ is the total reaction rate (i.e., total precipitated mass per unit of fluid volume and time), $\phi_{t o t}$ is the total porosity, $\phi_{t o t}=\phi_{i m}+\phi_{m}$, and $r_{m}$ and $r_{i m}$ are respectively the reaction rates in the mobile and immobile domain.

\subsection{Homogeneous medium}

In order to test the methodology, we compare the $f_{\text {mix }}$ estimates obtained with the proposed KDE method with an analytical solution. For this purpose, we consider a twodimensional homogeneous aquifer under steady-state uniform flow conditions. Solute transport is described by the traditional reactive advection-dispersion equation, without external forces or the presence of an immobile domain, i.e., $r_{i} \neq 0, f_{i}=0$ and $\Gamma_{i}=0$ in Eq. (51). Initially, the two aqueous species $\left\{c_{1}, c_{2}\right\}$ are in local equilibrium with the solid mineral such that $c_{1,0}=c_{2,0}$ (i.e., $u_{0}=0$ ). The initial equilibrium is 
then affected by a point-like instantaneous injection of water, that is still in chemical equilibrium with the mineral but with a different chemical composition. In this situation, knowing that $u=c_{1}-c_{2}$ is the conservative specie (De Simoni et al., 2005), the initial condition can be written as

$u\left(x, y, t=0^{+}\right)=u_{0}+\Delta u_{0} \delta\left(x-x_{0}\right) \delta\left(y-y_{0}\right)$,

where $\delta(\cdot)$ is the Dirac delta function, $\left(x_{0}, y_{0}\right)$ is the point of injection, and $\Delta u_{0}$ is the initial pulse of the $u$-concentration, the solution of the transport problem is (Bear, 1972)

$u(x, y, t)=u_{0}+\frac{\Delta u_{0}}{4 \pi t \sqrt{D_{L} D_{T}}} \exp \left(-\frac{\rho^{2}}{2}\right)$,

$\rho(x, y, t)=\sqrt{\frac{\left(x-x_{0}-v t\right)^{2}}{2 D_{L} t}+\frac{\left(y-y_{0}\right)^{2}}{2 D_{T} t}}$

where $D_{L}$ and $D_{T}$ are the longitudinal and transverse dispersion coefficient, and $v$ is the groundwater velocity. From Eq. (63), the analytical solution of the mixing term in Eq. (58) is
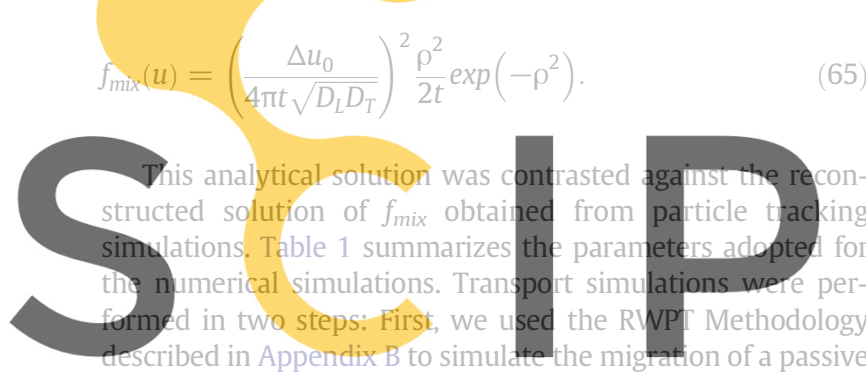

solute representing the component $u$; Then, we evaluated $f_{\text {mix. }}$

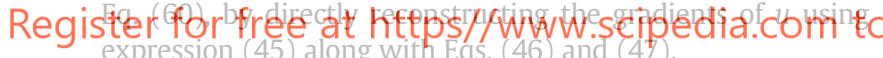

Transport simulations started with the injection of a large number of particles of equal mass, $m_{p}=\phi \Delta u_{0} / N_{p}$, at the point location $\left(x_{0}, y_{0}\right)$, being $N_{p}$ the total number of particles.

Table 1

Flow and transport parameters adopted during the numerical simulations performed in a homogeneous medium.

\begin{tabular}{ll}
\hline Parameter & Value \\
\hline Flow problem & \\
Number of cells in $x$ direction, $n_{x}$ & 160 \\
Number of cells in $y$ direction, $n_{y}$ & 160 \\
Cell size in $x$ direction, $\Delta x[L]$ & 1.0 \\
Cell size in $y$ direction, $\Delta y[L]$ & 1.0 \\
Reactive transport problem & \\
$x$ coordinate of injection, $x_{0}[L]$ & 20.0 \\
$y$ coordinate of injection, $y_{0}[L]$ & 80.0 \\
Injected mass, $M_{0}[M]$ & 1.0 \\
Darcy-Velocity, $q_{x}[L / T]$ & 0.3 \\
Darcy-Velocity, $q_{y}[L / T]$ & 0.0 \\
Porosity, $\phi[-]$ & 0.3 \\
Longitudinal dispersion, $D_{L}\left[L^{2} / T\right]$ & 5.0 \\
Transverse dispersion, $D_{T}\left[L^{2} / T\right]$ & 2.0 \\
Equilibrium constant $\left[M^{2} / L^{6}\right]$ & $10^{-7}$ \\
Pulse injection of $u, \Delta u_{0}\left[M / L^{6}\right]$ & 1.0 \\
Initial concentration of $u, u_{0}\left[M / L^{6}\right]$ & 0.0 \\
Random walk features & \\
Grid Courant number $[-]$ & 0.1 \\
\hline
\end{tabular}

Particle clouds were then measured at different times at which the reconstruction of $u$ and $f_{\text {mix }}$ was undertaken. The procedure used to reconstruct $f_{\text {mix }}$ based on the proposed KDE method is described in Appendix C.

Figs. 1 and 2 compare the analytical solution of $u$ and $f_{\text {mix }}$ at time $t=60$ with their corresponding optimal estimates obtained using the proposed KDE method. Simulations were performed with 2.5 million particles. The results are also contrasted against the traditional approach, which simply consists in counting particles within bins according to Eq. (12). In this approach, the gradients involved in $f_{\text {mix }}$ were computed using a finite difference spatial discretization of $u$ without any post-treatment. It is also compared to the coarsegraining technique, where the size of the bins was manually changed to obtain the most adequate smooth representation of $u$ and $f_{\text {mix }}$. In overall, figures show that the proposed KDE method is capable to automate the reconstruction of concentrations and reactions rates from particle distributions, and further generate a more superior depiction of the reactions rates compared with the traditional solution. The latter is seen by noticing that, for the same particle distribution, the KDE method is capable to provide a more complete reproduction of the reaction rates than that of the traditional approach obtained after coarse-graining, which still requires adding more particles into the system. The main difference stems from the incomplete reproduction of the maximum values of the reaction rates given

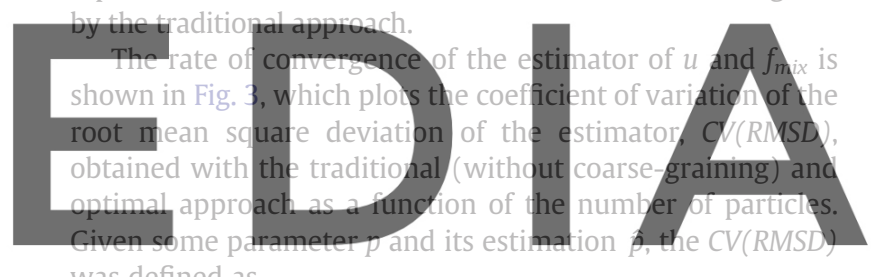

was defined as

\section{download the isfersion without the watermark} CV $($ RMSD $)=\frac{\bar{p}}{\bar{p}}$

where

$\bar{p}=\frac{1}{V} \int_{V} p(\mathbf{x}) d \mathbf{x}$,

$R M S D=\left(\frac{1}{V} \int_{V}(p(\mathbf{x})-\hat{p}(\mathbf{x}))^{2} d \mathbf{x}\right)^{1 / 2}$.

In applying the traditional approach, we fixed the size of the bin to $\Delta x=\Delta y=1$. Results show that the rate of convergence associated with the KDE method is much smaller than that of the corresponding traditional method. Thus, the $C V(R M S D)$ of concentrations displays a power law behavior with an exponent varying from -0.25 (KDE method) to -0.5 (traditional method). This is due to the fact that the use of an optimal support in the KDE method provides the "best unbiased" depiction of $u$ and $f_{\text {mix }}$ for any given number of particles, being the intersection between the traditional and optimal solution the number of particles needed in the traditional approach to obtain reliable predictions. Importantly, the traditional estimates of $f_{\text {mix }}$ are orders of magnitude larger than its corresponding optimal solution, meaning that an unfeasible number of particles is required when using the traditional method. 

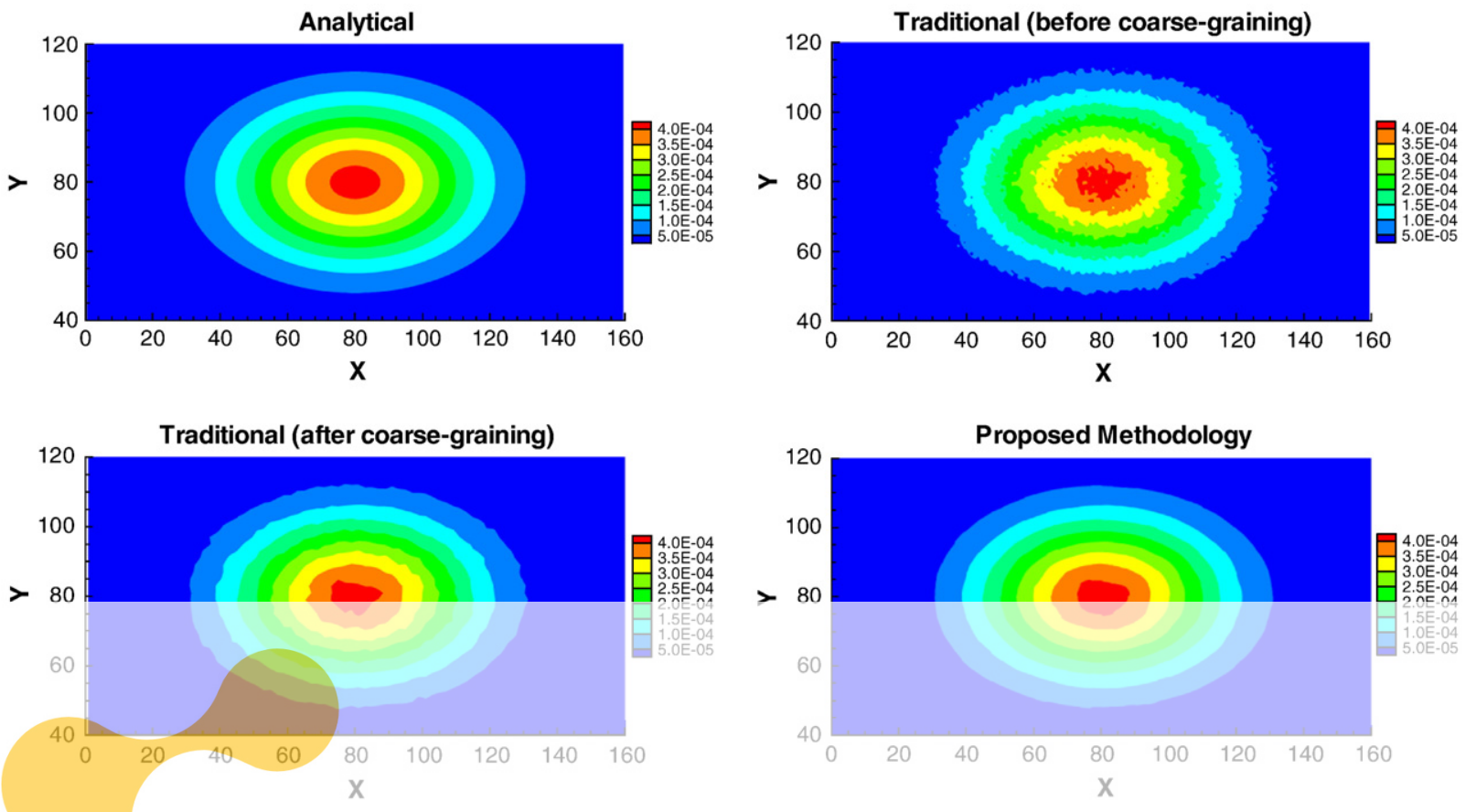

Fig. 1. Comparison of the analytical solution of $u$ with the traditional approach (before and after coarse-graining) and the proposed KDE methodology. The bin sizes

before and after coarse-graining were $\Delta x=\Delta y=1.0$ unit and $\Delta x=\Delta y=2.0$ units, respectively. Random walk simulations were performed with 2,500,000
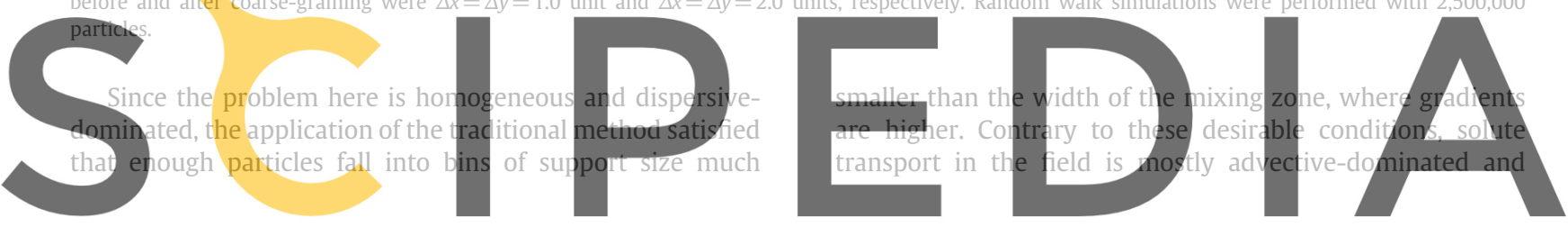

Analytical

Traditional (before coarse-graining)

Registerºr free at https//www.scipedia.com to download the version without the watermark
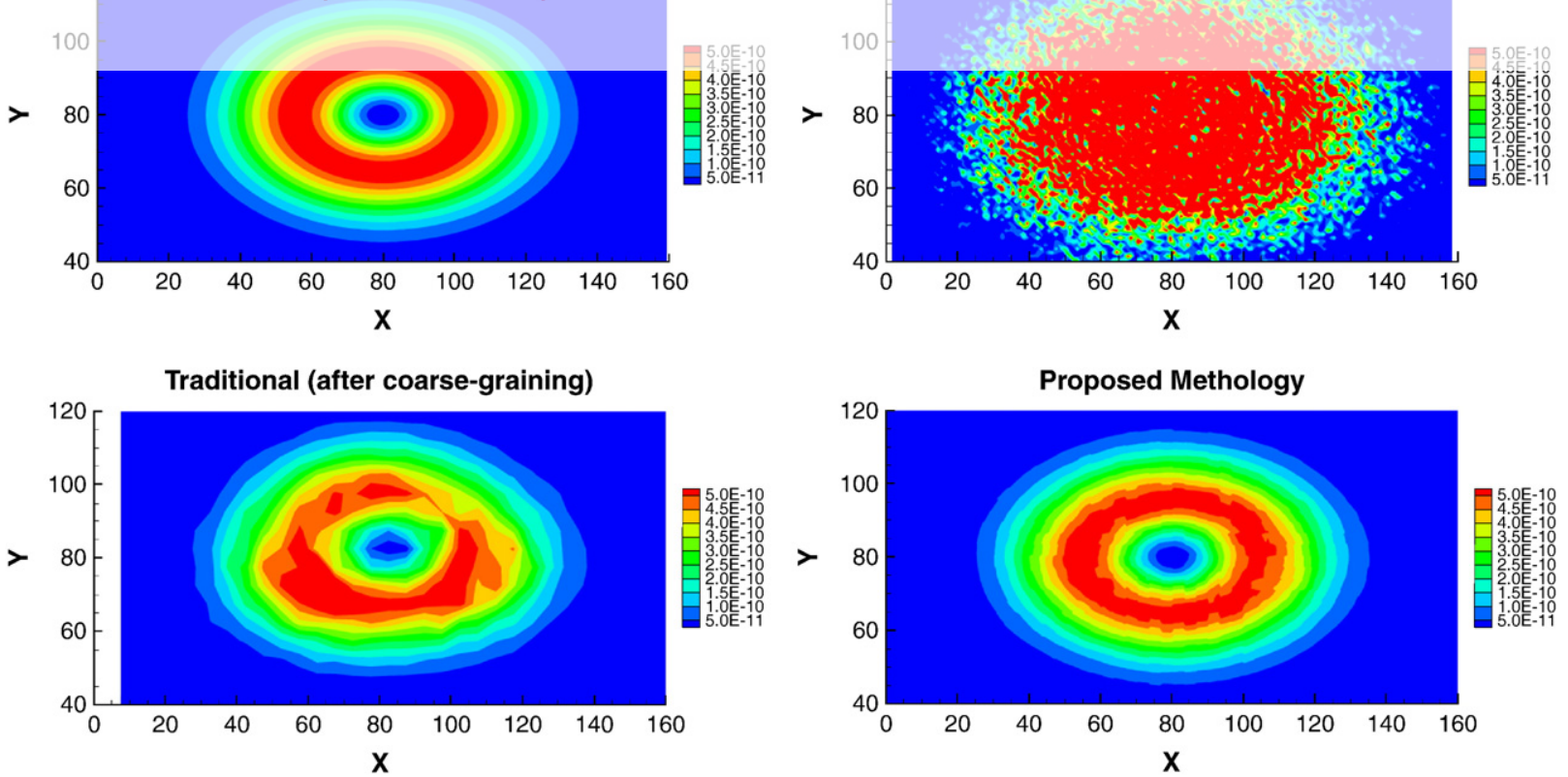

Fig. 2. Comparison of the analytical solution of $f_{\text {mix }}$ with the traditional approach (before and after coarse-graining) and the proposed KDE methodology at a given time $t=60$. The bin sizes before and after coarse-graining were $\Delta x=\Delta y=1.0$ unit and $\Delta x=\Delta y=4.5$ units, respectively. Random walk simulations were performed with 2,500,000 particles. 


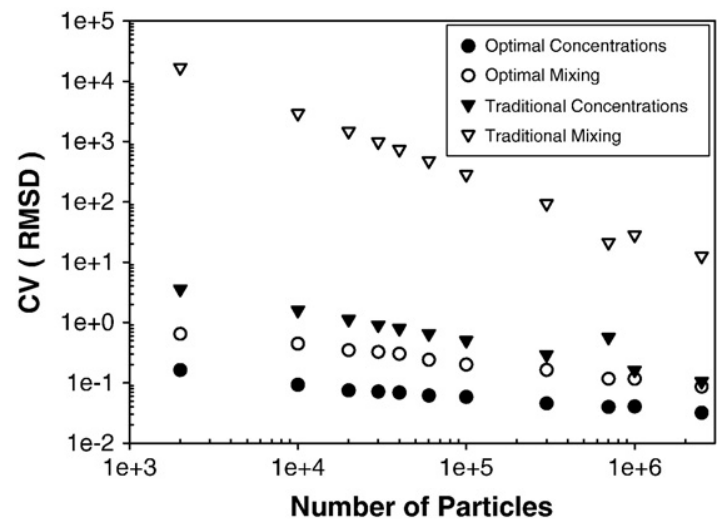

Fig. 3. Coefficient of variation of the root mean square deviation of the estimated concentration and mixing term obtained with the traditional and optimal approach as a function of the number of particles.

drastically influenced by heterogeneity. This produces highly distorted plumes with strong gradients and narrow mixing zones at the plume edges. Within this context, results indicate that the proposed KDE method to reconstruct reaction rates will allow the use of a more feasible number of particles to achieve the same quality of results.
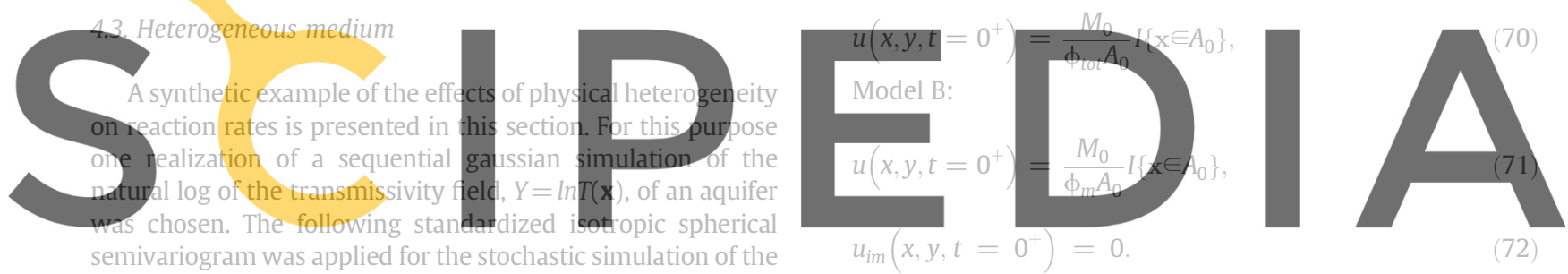

$u_{i m}\left(x, y, t=0^{+}\right)=0$.

$(72)$

$x=0$ and $x=160$ and with the no-flow at the remaining model boundaries.

Two conceptual transport models were considered: Solute transport in Model A was purely influenced by advection and dispersion $\left(r_{i} \neq 0\right.$ and $\left.\Gamma_{i}=0\right)$, whereas in Model B a mass sfer equation was added to the advection-dispersion $\left(r_{i} \neq 0\right.$ and $\left.r_{i} \neq 0\right)$. The parameters adopted for both simulations wer condured the migration of the conservative species, $u$ and $u_{i m}$, using the RWPT methodology described in Appendix B; Then, we evaluated $r_{m}$ and $r_{i m}$ through the expressions (57) and (58) by directly reconstructing the gradients of $u$ and $u_{i m}$ using the proposed KDE method.

In both models, the aquifer was initially in geochemical equilibrium at all points. A water with a different chemical composition was then injected instantaneously in a rectangular area $A_{0}$ of 30 units width and 50 units height located orthogonal to the principal flow direction. A sketch of the setup adopted for the transport simulations is provided in Fig. 4. Knowing again that $u=c_{1}-c_{2}$ and $u_{i m}=c_{1, i m}-c_{2, i m}$ are the conservative species for this problem, the initial condition can be written in terms of $u$ and $u_{i m}$ as

Model A:
$\frac{\gamma(h)}{\sigma_{Y}^{2}}= \begin{cases}1.5\left(\frac{h}{a}\right)-0.5\left(\frac{h}{a}\right)^{3} & \text { if } h \leq a \\ 1 & \text { otherwise }\end{cases}$

where $a[L]$ is the range, $h$ is the lag distance, and $\sigma_{Y}^{2}$ is the variance of $\ln T$ (see Table 2). The computational domain represents a squared aquifer with dimensions of $L_{x}=160$ and $L_{y}=160$, and a discretization of $\Delta x=\Delta y=1.0$. The aquifer was assumed to be confined and with constant head boundaries at

\section{Table 2}

Flow problem parameters adopted during the numerical simulations performed in a heterogeneous medium.

\begin{tabular}{ll}
\hline Parameter & Value \\
\hline Flow problem & \\
Number of cells in $x$ direction, $n_{x}$ & 160 \\
Number of cells in $y$ direction, $n_{y}$ & 160 \\
Cell size in $x$ direction, $\Delta x[L]$ & 1.0 \\
Cell size in $y$ direction, $\Delta y[L]$ & 1.0 \\
Mean hydraulic gradient in $x$ direction, $J_{x}[-]$ & 0.001 \\
Mean hydraulic gradient in $y$ direction, $J_{y}[-]$ & 0.0 \\
Heterogeneous field & \\
Geometric mean of T $\left[L^{2} / T\right]$ & 1.0 \\
Variance of ln T [-] & 3.6 \\
Range of variogram $[L]$ & 16.0 \\
\hline
\end{tabular}

Table 3

Transport parameters adopted during the numerical simulations performed in a heterogeneous medium.

\begin{tabular}{lll}
\hline Parameter & Model A & Model B \\
\hline Area of injection, $A_{0}\left[L^{2}\right]$ & $30 \times 50$ & $30 \times 50$ \\
Injected mass, $M_{0}[M]$ & 0.3 & 0.15 \\
Mobile porosity, $\phi[-]$ & 0.3 & 0.15 \\
Immobile porosity, $\phi[-]$ & 0. & 0.15 \\
Longitudinal dispersion, $D_{L}\left[L^{2} / T\right]$ & 0.3 & 0.3 \\
Transverse dispersion, $D_{T}\left[L^{2} / T\right]$ & 0.1 & 0.1 \\
Mass transfer coefficients, $\alpha_{j}\left[T^{-1}\right]$ & 0. & 0.0002 \\
Equilibrium constant $\left[M^{2} / L^{6}\right]$ & $10^{-7}$ & $10^{-7}$ \\
Constant time step $[T]$ & 7. & 7. \\
Number of particles $[-]$ & $2.5 \times 10^{6}$ & $5.0 \times 10^{6}$ \\
\hline
\end{tabular}




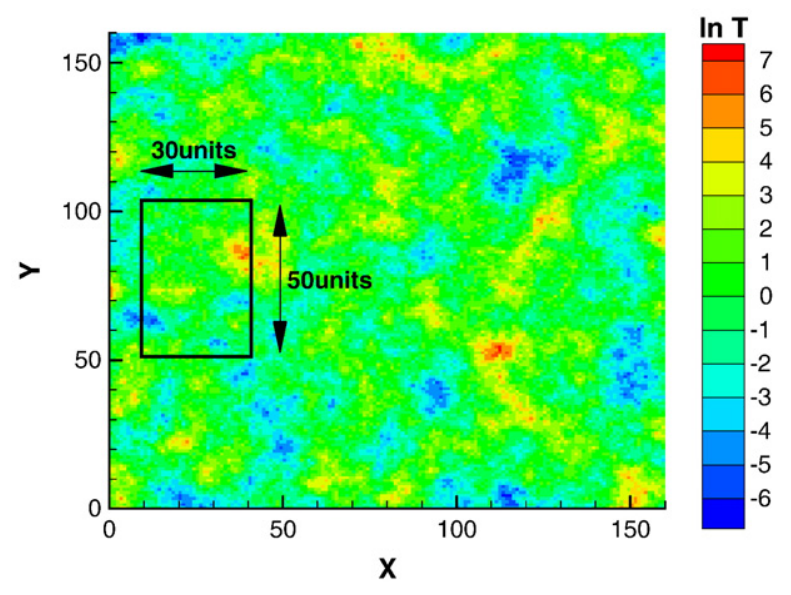

Fig. 4. Design of transport numerical simulations.

$f_{\text {mix }}$ and chemical speciation $f_{\text {chem }}$, Eqs. (60) and (59), are also depicted. At this particular time, transverse dispersion has still not induced complete mixing, and the shape of the plume of the conservative component $u$ is mostly distorted according to the velocity field. This produces sharp fronts and narrow mixing areas at the plume edges. Consistent with this picture, the proposed KDE method predicts chemical reactions to take place mostly at the plume boundaries. By looking
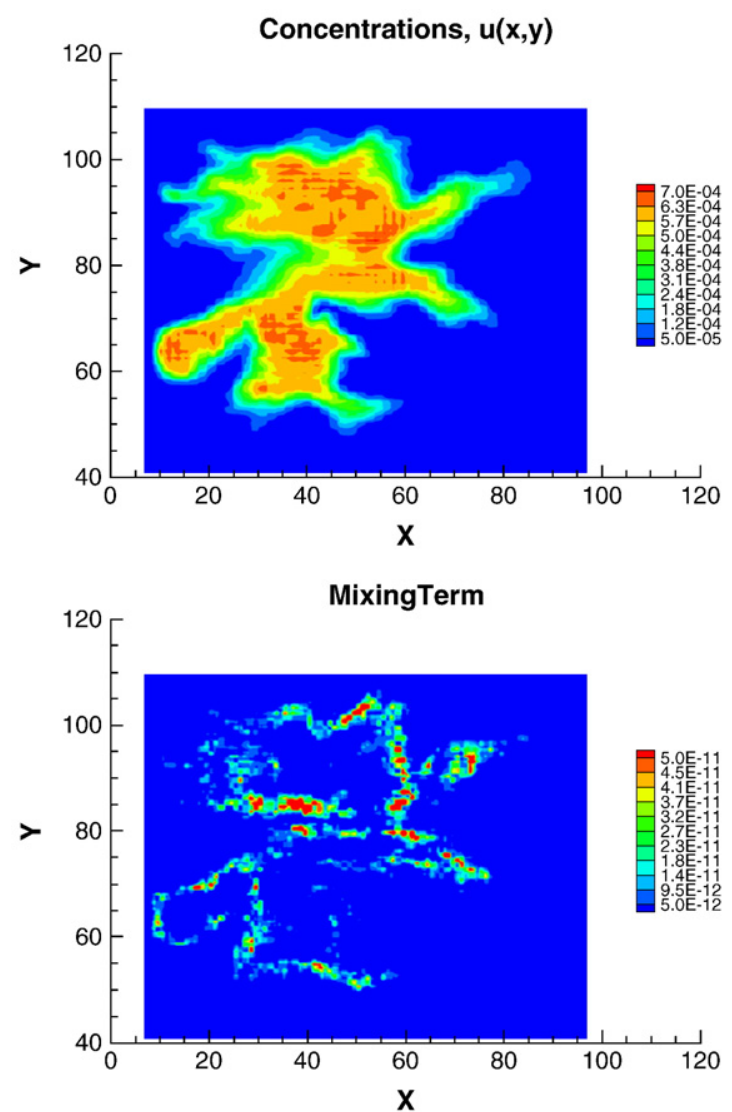

at the mixing and chemistry terms, we see that in this particular case, the chemistry term mainly serves to amplify the mixing impulse without significantly changing the location of the hot spots of the reaction rates.

Most frequently in stochastic contaminant hydrology, the spatial fluctuations of the dispersion tensor due to the randomness of the velocity field are neglected and considered of minor importance. That is to say that the dispersion tensor in the conceptual transport model is assumed constant and fixed to some averaged value. This assumption may not be valid in reactive transport problems where the processes taking place at the local scale can still influence the global behavior (Fernàndez-Garcia et al., 2008). In order to assess the effects of assuming a constant dispersion during the calculation of the reaction rate, we estimated $f_{\text {mix }}$ in two different ways:

$$
\begin{aligned}
& \phi_{m} f_{\text {mix }}(\mathbf{x}, t)=\alpha_{L}\|q(\mathbf{x}, t)\|\left(\frac{\partial u}{\partial x}\right)^{2}+\alpha_{T}\|q(\mathbf{x}, t)\|\left(\frac{\partial u}{\partial y}\right)^{2} \\
& \phi_{m} f_{\text {mix }}(\mathbf{x}, t)=\bar{D}_{L}\left(\frac{\partial u}{\partial x}\right)^{2}+\bar{D}_{T}\left(\frac{\partial u}{\partial y}\right)^{2}
\end{aligned}
$$

where to make results comparable we have selected the constant diffusion parameters so that

$\bar{D}_{L}=\alpha_{L} \bar{q} \quad \bar{D}_{T}=\alpha_{T} \bar{q}$
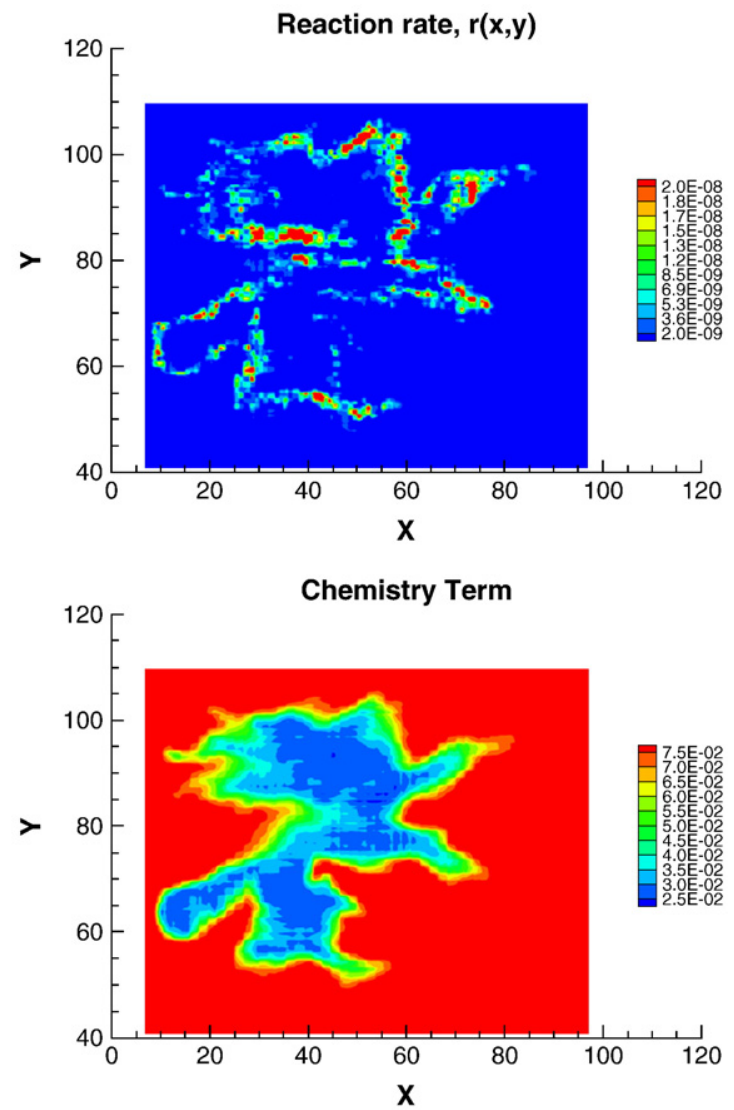

Fig. 5. Numerical simulations of concentrations and reactions rates in a heterogeneous aquifer at time $t=4064$. Random walk simulations were performed with $2,500,000$ particles. 

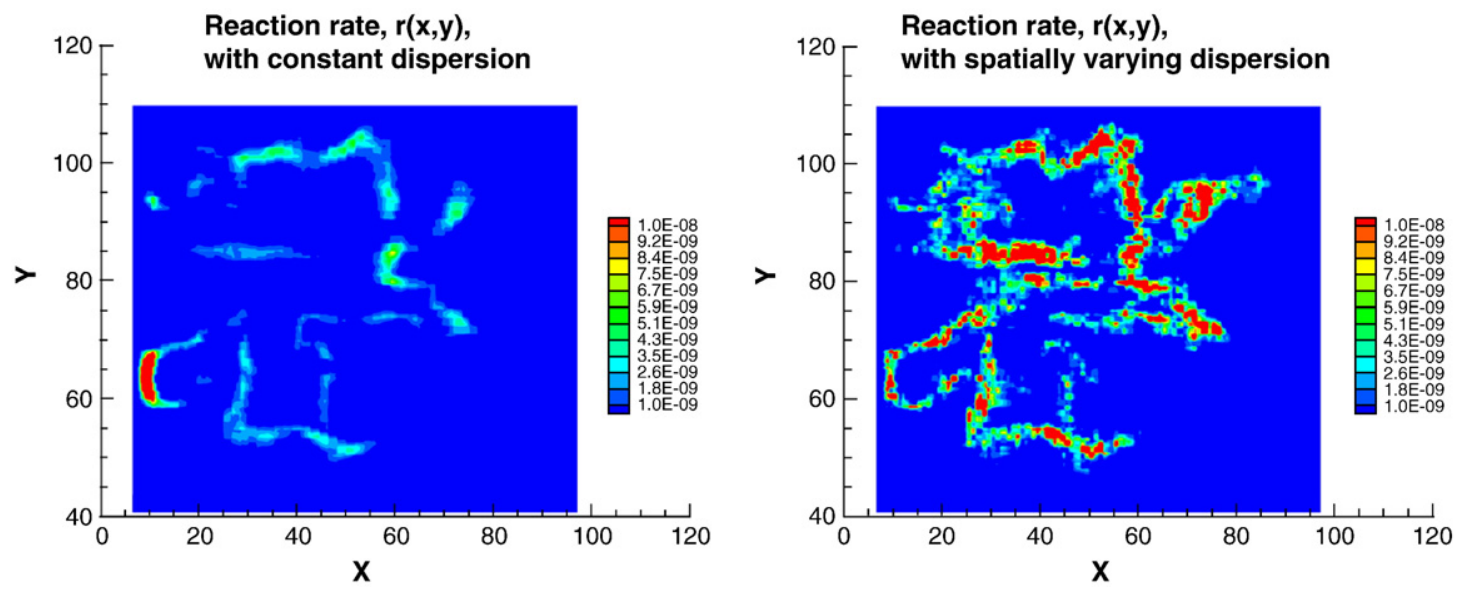

Fig. 6. Comparison of the reactions rates obtained in a heterogeneous aquifer using constant dispersion coefficients with those obtained using spatially varying dispersion coefficients at time $t=4064$. The constant dispersion coefficients were computed using an average of the absolute value of the point velocities. Random walk simulations were performed with 2.5 million particles.

with

$\bar{q}=\sqrt{\bar{q}_{x}^{2}+\bar{q}_{y}^{2}}, \bar{q}_{i}=\frac{1}{V} \int_{V}\left|q_{i}(\mathbf{u})\right| d \mathbf{u}, i=1,2$,

being $V$ the area of the domain. The reaction rates simulated using a constant or a spatially varying dispersion tensor are
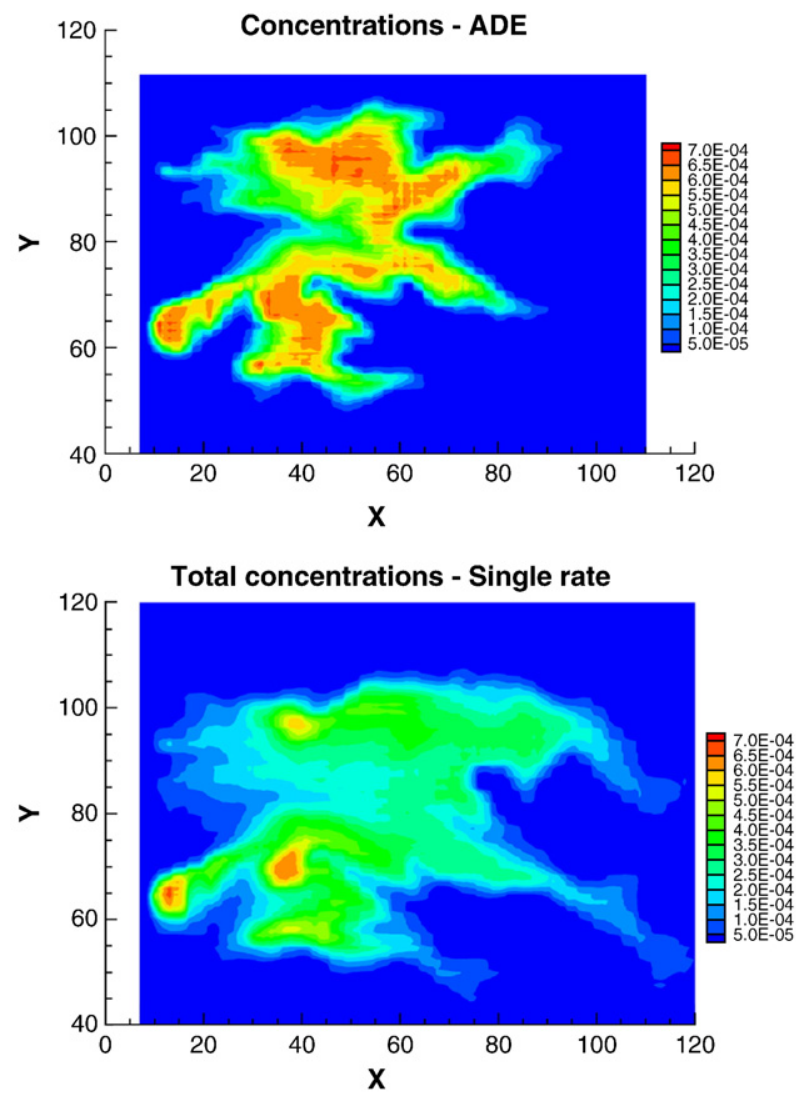

shown in Fig. 6. Remarkably, a constant dispersion tensor significantly oversmoothed the reaction rates, concentrating their maximum values nearby the source where concentration gradients are higher. This is in contrast to the reaction rates obtained using a spatially varying dispersion tensor which showed larger reaction rates at the leading front of the plume. Recalling that mixing is caused by the joint effect of dispersion
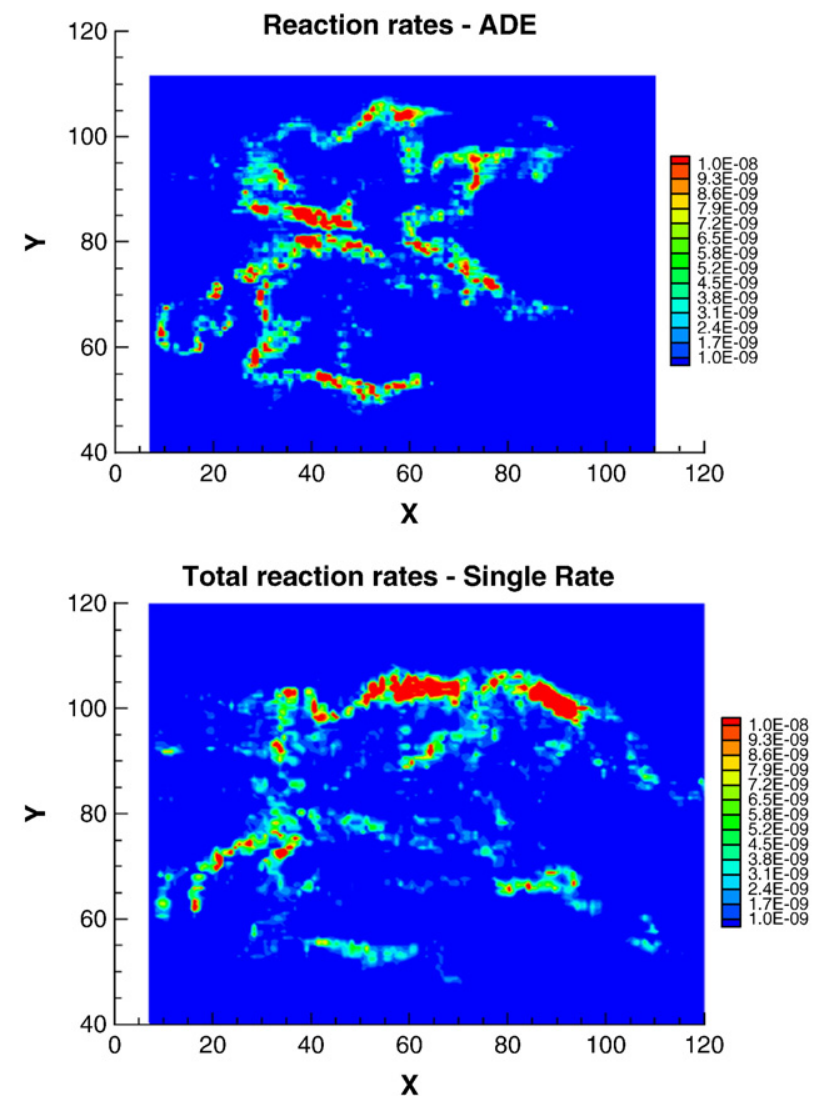

Fig. 7. Comparison of the reactions rates of the single-rate mass transfer model with the ADE model at time $t=5410$. The total concentrations and reaction rates associated with the single-rate mass transfer model include both the mobile and the immobile contribution. 
and concentration gradients, see Eq. (5), we attribute this behavior to the fact that the plume front is moving preferentially through areas of high velocities, where the effects of velocitydriven dispersion and large concentration gradients coexist. These effects are seen remarkably more important in the transverse direction to the mean flow direction.

The choice of a proper transport model may also drastically influence the model predictions of reaction rates. This is shown in Fig. 7, which compares the simulated reactions rates produced by models A and B at time $t=5410$. Interestingly, the addition of a mass transfer term into the advection-dispersion equation enhances the speed of dilution into the system. This is due to the joint effect of local mass transfer processes, intrinsically described by the transport model through $\Gamma_{i}(\mathbf{x}, t)$ (Donado et al., 2009), and physical heterogeneity, i.e., the spatial variability of $q(\mathbf{x})$ in the governing transport equation.

\section{Conclusions}

The inherent fluctuations associated with the estimates of concentrations and their functionals (reaction rates) have typically prevented the use of particle tracking techniques to simulate complex reactive problems. In this paper, we have shown that this appreciation stems from a naive understanding of the reconstruction of functionals of concentrations in the particle tracking literature. Based on kernel density functions, we have presented an efficient method for the reconstruction of the reactions rates of chemical species from particle distributions. In doing this, the following main findings should be highlighted:

1. The reconstruction of reaction rates or other functionals of species concentrations in multi-dimensional reactive transport problems can easily be optimized and further automated via KDE methods. This provides a powerful tool for all branches of particle tracking techniques which require generating continuous fields of a system variable from discrete particle distributions. Thus, this method is well suited to directly reconstruct important system variables such as the dilution index $E$, the destruction of the concentration variance $f_{\sigma_{c}^{2}}$, the destruction/production of uncertainty $f_{p d f}$, the reaction rates of reactive species $r$, boundary mass fluxes and others.

2. Since the proposed KDE method avoids the propagation of the inherent fluctuations associated with the estimates of concentrations and their functionals, it renders particle tracking techniques the capability to be potentially coupled to any existing geochemical transport code. In this regard, we have shown that the coupling will be most efficient if the conceptual framework of De Simoni et al., 2005, which deconstructs the reactive problem into a conservative one plus speciation, is adopted to either calculate chemical species concentrations or their reaction rates.

The proposed KDE method was then used to analyze particle tracking simulations performed in a highly heterogeneous aquifer with the objective to evaluate the reactions rates of a bimolecular precipitation/dissolution chemical system. Two different transport conceptual models were considered: the traditional advection-dispersion model and the single-rate mass transfer model. The main results can be summarized as follows:

1. The proposed KDE method allows the reconstruction of sharp plume gradients and narrow mixing zones in heterogeneous aquifers, which are otherwise not well estimated by traditional approaches.

2. Simulation results show that the spatial variability of the dispersion tensor is crucial for making predictions of the reaction rates of chemical species taking place in heterogeneous aquifers. A constant dispersion tensor produces smoother representations of the reaction rate with a less amount of total precipitate. The reason is that the mixing term, $f_{\text {mix }}$, depends on the balance of two driven forces: velocity-driven dispersion and concentration gradients. At short times, the gradients close to the source are extremely large and control the reaction rate. As times evolve, the gradients slowly dissipate and compete with the velocitydriven dispersive processes. In this regime, the fact that the plume front moves preferentially through high velocities areas creates strong reaction rates at the leading edges of the plume. These effects were observed to be more important in the transverse direction to the mean flow direction.

3. The choice of the transport model can drastically effect mixing-driven chemical reactions. In this context, the joint effect of local mass transfer processes (intrinsically described in the transport model) and heterogeneity is shown to substantially enhanced mixing. At large times, when the plume is highly diluted (small concentration gradients), the reaction rate is mainly controlled by the transverse dispersion processes taking place at the leading front of the plume.

\section{Acknowledgments}

The authors acknowledge the financial support provided by ENRESA, by the Agència de Gestió d'Ajuts Universitaris i de Recerca of the Catalan Government, and by the Spanish CICYT through projects RARAVIS (CGL2009-11114), HEROS (CGL200766748), and SCARCE (CONSOLIDER).

\section{Appendix A. Statistics of the estimators of concentrations and their derivatives}

This Appendix describes the statistics of the estimates of univariate probability density functions and their derivatives. This is illustrated by actually looking at the estimates of $p(t)$, i.e., the normalized flux concentrations. Let us consider a random sample $T_{1}, \ldots, T_{N_{a}}$ of $N_{a}$ particle arrival times observed at a control location. Each random sample is described by the same probability density function $p(t)$, and assumed independent. This is a valid assumption in most random walk codes because the simulation of $N_{a}$ particles starts from $N_{a}$ independent uniformly (or normally) distributed numbers. We will also consider that all particles carry the same mass $m_{p}$. The kernel estimator of $\mathrm{d}^{k} p / \mathrm{d} t^{k}$ is

$$
\hat{p}^{(k)}(t) \equiv \frac{1}{H^{1+k} M_{a}} \sum_{p} m_{p} K_{k}\left(\frac{t-T_{p}}{H}\right) .
$$


Optimal estimates of $p(t)$ can be determined by minimizing the Mean Integrated Square Error function (MISE), Eq. (22). MISE can be expressed as the sum of the integrated squared bias and the integral of variance through Eq. (23). We will start by evaluating the integrated squared bias. Taking the expected value of Eq. (76) we have that

$E[\hat{p}(t)]=\frac{1}{H^{1+k}} E\left\{K_{k}\left(\frac{t-T_{p}}{H}\right)\right\}$.

By the definition of the expected operator, this can be written as

$E[\hat{p}(t)]=\frac{1}{H^{1+k}} \int K_{k}\left(\frac{t-\tau}{H}\right) p(\tau) d \tau$,

Using then a change of variable $s=(t-\tau) / H$ yields

$E[\hat{p}(t)]=\frac{1}{H^{k}} \int K_{k}(s) p(t-s H) d s$,

which expresses that the expected value is given by the convolution of $K_{k}$ with $p$. Using a Taylor expansion of $p$ around $t$,

$p(t+s H)=\sum_{n=0}^{\infty} \frac{1}{n !} p^{(n)}(t)(-1)^{n} s^{n} H^{n}$,

and knowing that $K_{k}$ satisfies Eq. (43) yields

$E[\hat{p}(t)]=p^{(k)}(t)+\frac{H^{m-k}}{m !} p^{(m)}(t) \mu_{m}\left(K_{k}\right)+O\left(H^{m-k}\right)$,

so that the local squared bias of the estimator, $\left(E\left[\hat{p}^{(k)}\right]-p^{(k)}\right)^{2}$, is

$\operatorname{Bias}^{2}\left[\hat{p}^{(k)}(t)\right]=\frac{H^{2(m-k)}}{m !^{2}}\left(p^{(m)}(t)\right)^{2}\left(\mu_{m}\left(K_{k}\right)\right)^{2}+O\left(H^{2(m-k)}\right)$.

On the other hand, the integral of the variance can be estimated as follows. Knowing that $M_{a}=N_{p} m_{p}$ and taking the variance of the estimator of $\hat{p}(t)$ we obtain

$\operatorname{Var}[\hat{p}(t)]=\frac{1}{H^{2(1+k)} N_{p}} \operatorname{Var}\left\{K_{k}\left(\frac{t-T_{p}}{H}\right)\right\}$,

where we have used that the random samples of particle arrival times are independent random variables. Knowing that $\operatorname{Var}\left[K_{k}\right]=E\left[K_{k}^{2}\right]-\left(E\left[K_{k}\right]\right)^{2}$ and using the change of variable $s=(t-\tau) / H$ yields

$\operatorname{Var}\left\{K_{k}\right\}=\int K_{k}^{2}(s) p(t-s H) H d s-\left(\int K_{k}(s) p(t-s H) H d s\right)^{2}$.

Using a Taylor expansion of $\hat{p}^{(k)}$ around $t$ and knowing that $K_{k}$ satisfies Eq. (43) yields

$\operatorname{Var}\left\{K_{k}\right\}=H R\left(K_{k}\right) p(t)-H^{2(1+k)}\left(p^{(k)}(t)\right)^{2}+\ldots,(85)$ and therefore, substituting Eq. (85) into Eq. (83), leads to

$$
\begin{aligned}
\operatorname{Var}[\hat{p}(t)]= & \frac{1}{N_{p} H^{2 k+1}}\left(R\left(K_{k}\right) p(t)-H^{2 k+1} p^{2}(t)\right) \\
& +O\left(\left(N_{p} H^{2 k+1}\right)^{-1}\right) .
\end{aligned}
$$

Substituting Eqs. (82) and (86) into Eq. (23), and taking the limit of MISE as $N_{p} H \rightarrow \infty$ and $H \rightarrow 0$, the following asymptotic value is obtained

$$
\operatorname{AMISE}(H)=\frac{H^{2(m-k)}}{m !^{2}} R\left(p^{(m)}(t)\right)\left(\mu_{m}\left(K_{k}\right)\right)^{2}+\frac{1}{N_{p} H^{2 k+1}} R\left(K_{k}\right) .
$$

\section{Appendix B. Algorithm to simulate transport with the random walk methodology}

This Appendix describes the algorithm used to simulate the transport of the conservative species, $u$ and $u_{i m}$, with the random walk particle tracking methodology. The displacement of a particle is given by a drift term that related to the advective movement and a superposed Brownian motion responsable for dispersion,

$$
\begin{aligned}
\mathbf{X}_{p}(t+\Delta t)= & \mathbf{X}_{p}(t)+\int_{t}^{t+\Delta t} \mathbf{A}\left(\mathbf{X}_{p}, \tau\right) d \tau \\
& +\mathbf{B}\left(\mathbf{X}_{p}, t\right) \Delta \mathbf{W}_{t},
\end{aligned}
$$

where $\Delta t$ is the time step, $\mathbf{X}_{p}(t)$ is the position of a particle at time $t, \mathbf{A}$ is a drift vector, i.e., any change in $E\left[\mathbf{X}_{p}(t)\right]$ is due to the drift term, and $\mathbf{B} \Delta \mathbf{W}_{\tau}$ is the noise term. The tensor $\mathbf{B}$ is the displacement matrix that determines the strength of the particle random motion, and $\mathbf{W}_{t}$ is an $n$ variable Wiener process determined by

$\mathbf{B}\left(\mathbf{X}_{p}, t\right) \triangle \mathbf{W}_{\tau}=\mathbf{B}\left(\mathbf{x}_{p}, t\right) \xi(t) \sqrt{\Delta t}$

where $\xi(t)$ is a vector of independent (uncorrelated in space and time), normally distributed random variables with zero mean and unit variance.

Itô (1951) demonstrated that the particle density distribution $f\left(\mathbf{X}_{p}, t\right)$, defined as the probability of finding a particle within a given interval $\left[\mathbf{X}_{p}, \mathbf{X}_{p}+\mathrm{d} \mathbf{X}_{p}\right]$ at a given time $t$, obtained from Eq. (88) fulfills, in the limit of large particle numbers and an infinitesimally small step size, the FokkerPlanck equation. This partial differential equation is similar but not equal to the ADE. An analogy between them is established by the following relationship

$$
\begin{aligned}
& \mathbf{A}=\frac{\mathbf{q}}{\phi R}+\frac{1}{\phi R} \nabla \cdot(\phi \mathbf{D}) \\
& 2 \frac{\mathbf{D}}{R}=\mathbf{B} \cdot \mathbf{B}^{t}
\end{aligned}
$$

In the numerical simulations, the velocity field needed to simulate transport was obtained by previously solving the flow problem using a finite difference code, MODFLOW2000 (Harbaugh et al., 2000). This velocity field was then incorporated into 
a random walk code, RW3D-MRMT (Fernàndez-Garcia et al., 2005; Salamon et al., 2006b) to solve the transport problem. In all cases, the same mass was associated to all particles, $m_{p}=M_{0} / N_{p}$, and the time integration of the drift term in Eq. (88) was calculated using the semi-analytical tracking method of Pollock (1988).

RW3D-MRMT simulates the single-rate mass transfer model by simply tracking the state of a particle (Salamon et al., 2006b). The state of a particle is an attribute that defines the domain at which the particle is present at a given time within the double porosity medium. The change from one state to another is easily determined using transition probabilities. The transition probability, $P_{i j}(\Delta t)$, that a particle presently in state $i$ will be in state $j$ at a time $t+\Delta t$ is given by

$$
\mathbf{P}(\Delta t)=\left(\begin{array}{cc}
\frac{1+\beta e^{-(1+\beta) \alpha \Delta t}}{1+\beta} & \frac{1-e^{-(1+\beta) \alpha \Delta t}}{1+\beta} \\
\frac{\beta-\beta e^{-(1+\beta) \alpha \Delta t}}{1+\beta} & \frac{\beta+e^{-(1+\beta) \alpha \Delta t}}{1+\beta}
\end{array}\right)
$$

If a particle is in the mobile domain, then it is susceptible to advection and dispersion, otherwise the particle is not allowed to move. Having calculated the phase transition probabilities, numerical implementation into particle tracking is done easily. For each time step a uniform $[0,1]$ random number $Y$ is drawn for each particle and is compared to the corresponding probability. The state of a particle being in the mobile phase is adjusted accordingly.

\section{Appendix C. Algorithm to reconstruct mixing and reaction rates}

The algorithm used to directly estimate $f_{\text {mix }}$ and $r$ from particle clouds representing $u$ during numerical simulations proceeds as follows: (1) Discretized the $x$ and $y$ axes in bins $\left\{B_{x_{i}}\right\}$ and $\left\{B_{y_{j}}\right\}$; (2) Estimate the marginal densities $p(x)$ and $p(y)$ and their derivatives using univariate kernel estimators with an optimal support; (3) Estimate the conditional densities $p\left(x \mid y \in B_{y_{j}}\right)$ and $p\left(y \mid x \in B_{x_{i}}\right)$ and their derivatives for all $i, j$ using univariate kernel estimators with an optimal support; (4) Reconstruct the partial derivatives of the bivariate probability density function based on Eqs. (46) and (47); (5) Calculate the gradients of $u$ by means of Eq. (45); and (6) Estimate $f_{\text {mix }}$ and $r$ by using Eqs. (60) and (61), respectively.

The optimal bandwidth of the estimates of the probability density function and their derivatives were computed through Eqs. (28) and (44) by employing Gaussian Kernel functions. In doing this, the plug-in method of (Engel et al., $1994)$ was chosen to estimate the corresponding $L^{2}$-norm, $R$ $\left(p^{(m)}\right)$, involved in Eqs. (28) and (44).

\section{References}

Arulampalam, M.S., Maskell, S., Gordon, N., Clapp, T., 2002. A tutorial on particle filters for online nonlinear/non-Gaussian Bayesian tracking. IEEE TTrans. Signal Process. 50 (2), 174-188.

Bear, J., 1972. Dynamics of Fluids in Porous Media. Elsevier, New York.

Bellin, A., Tonina, D., 2007. Probability density function of non-reactive solute concentration in heterogeneous porous formations. J. Contam. Hydrol. 94 (1-2), 109-125.
Benson, D.A., Wheatcrat, S.W., Meerschaert, M.M., 2000. Application of a fractional advection-dispersion equation. Water Resour. Res. 36 (6), 1403-1412.

Berkowitz, B., Cortis, A., Dentz, M., Scher, H., 2006. Modeling non-fickian transport in geological formations as a continuous time random walk. Rev. Geophys. 44. doi:10.1029/2005RG000178 RG2003.

Carrera, J., Sánchez-Vila, X., Benet, I., Medina, A., Galarza, G., Guimerà, J., 1998. On matrix diffusion: formulations, solution methods and qualitative effects. Hydrogeol. J. 6, 178-190.

Chang, Ch., Ansari, R., 2005. Kernel particle filter for visual tracking. IEEE Signal. Process. Lett. 12 (3), 242-245.

Dentz, M., Berkowitz, B., 2003. Transport behavior of a passive solute in continuous time random walks and multirate mass transfer, Water Resour. Res 39 (5), 1111. doi:10.1029/2001WR001163.

De Simoni, M., Carrera, J., Sanchez-Vila, X., Guadagnini, A., 2005. A procedure for the solution of multicomponent reactive transport problems. Water Resour. Res. 41, W11410. doi:10.1029/2005WR004056.

Donado, L. D., X. Sanchez-Vila, M. Dentz, J. Carrera, and D. Bolster (2009), Multicomponent reactive transport in multicontinuum media, Water Resour. Res., doi:10.1029/2008WR006823, in press.

Duong, T., Hazelton, M.L., 2003. Plug-in bandwidth matrices for bivariate kernel density estimation. Nonparametric Stat. 15 (1), 17-30.

Duong, T., Cowling, A., Koch, I., Wand, M.P., 2008. Feature significance for multivariate kernel density estimation. Comput. Stat. Data Anal. 52, 4225-4242.

Engel, J., Herrmann, E., Gasser, T., 1994. An iterative bandwidth selector for kernel estimation of densities and their derivatives. Nonparametric Stat. 4, 21-34.

Fernàndez-Garcia, D., Illangasekare, T.H., Rajaram, Harihar, 2005. Differences in the scale dependence of dispersivity estimated from temporal and spatial moments in chemically and physically heterogeneous porous media. Adv. Water Resour. 28, 745-759.

Fernàndez-Garcia, D., Sanchez-Vila, X., Guadagnini, A., 2008. Reaction rates and effective parameters in stratified aquifers. Adv. Water Resour. 31, 1364-1376.

Fernàndez-Garcia, D., Llerar-Meza, G., Gómez-Hernández, J.J., 2009. Upscaling transport with mass transfer models: mean behavior and propagation of uncertainty. Water Resour. Res. 45, W10411. doi:10.1029/2009WR007764.

Fiorotto, V., Caroni, E., 2002. Solute concentration statistics in heterogeneous aquifers for finite Peclet values. Transp. Porous Media 48 (3), 331-351.

Gillespie, D., 1977. Exact stochastic simulation of coupled chemical reactions. J. Phys. Chem. 81 (25), 2340-2361.

Haggerty, R., Gorelick, S.M., 1995. Multiple-rate mass transfer for modeling diffusion and surface reactions in media with pore-scale heterogeneity. Water Resour. Res. 31 (10), 2383-2400.

Haggerty, R., McKenna, S.A., Meigs, L.C., 2000. On the late-time behaviour of tracer test breakthrough curves. Water Resour. Res. 36 (12), 3467-3479.

Harbaugh, A.W., Banta, E.R., Hill, M.C., McDonald, M.G., 2000. MODFLOW-2000. The U.S. Geological Survey Modular Ground-Water Model-user guide to modularization concepts and the ground-water flow process, Open-file Report. 00-92.

Hardle, W., 1990. Smoothing Techniques with Implementation in S. Springer-Verlag, New York.

Herrera, P.A., Massabó, M., Beckie, R., 2008. A meshless method to simulate solute transport in heterogeneous porous media. Adv. Water Resour. 32 (3), 413-429.

Itô, K., 1951. On Stochastic Differential Equations.Vol. 4. Am. Math. Soc, New York, pp. 289-302.

Jones, M.C., Marron, J.S., Sheater, S.J., 1996. A brief survey of bandwidth selection for density estimation. J. Am. Stat. Assoc. 91, 401-407.

Kapoor, V., Gelhar, L.W., 1994. Transport in three-dimensionally heterogeneous aquifers. 1. Dynamics of concentration fluctuations. Water Resour. Res. 30 (6), 1775-1788.

Kapoor, V., Kitanidis, P.K., 1998. Concentration fluctuations and dilution in aquifers. Water Resour. Res. 34 (5), 1181-1193.

Kitanidis, P.K., 1994. The concept of the dilution index, Water Resour. Res., 30 (7), 2011-2026.

Marron, J.S., Nolan, D., 1989. Canonical kernels for density estimation. Stat. Probab. Lett. 7, 195-199.

Neuman, S.P., Tartakovsky, D.M., 2008. Perspective on theories of non-Fickian transport in heterogeneous media. Adv. Water Res. 5, 670-680. doi:10.1016/j.advwatres.2008.08.005.

Park, B.U., Marron, J.S., 1990. Comparison of data-driven bandwidth selectors. J. Am. Stat. Assoc. 85 (409), 66-72.

Pollock, D.W., 1988. Semianalytical computation of path lines for finite difference models. Ground Water 26 (6), 743-750.

Riva, M., Guadagnini, A., Fernàndez-Garcia, D., Sanchez-Vila, X., Ptak, T., 2008. Relative importance of geostatistical and transport models in describing heavily tailed breakthrough curves at the Lauswiesen site. J. Contam. Hydrol. 101, 1-13. 
Sain, S.R., 2002. Multivariate locally adaptive density estimation. Comput. Stat. Data Anal. 39, 165-186.

Salamon, P., Fernàndez-Garcia, D., Gómez-Hernández, J.J., 2006a. A review and numerical assessment of the random walk particle tracking method. J. Contam. Hydrol. 87, 277-305.

Salamon, P., Fernàndez-Garcia, D., Gómez-Hernández, J.J., 2006b. Modeling mass transfer processes using random walk particle tracking. Water Resour. Res. VOL.42, W11417. doi:10.1029/2006WR004927 2006

Sanchez-Vila, X., Guadagnini, A., Fernàndez-Garcia, D., 2009. Conditional probability density functions of concentrations for mixing-controlled reactive transport in heterogeneous aquifers. Math. Geosci. 41, 323-351.

Silverman, B., 1986. W. Chapman and Hall, Density Estimation for Statistics and Data Analysis, London.

Simonoff, J., 1995. A simple, automatic and adaptive bivariate density estimator based on conditional densities. Stat. Comput. 5, 245-252.

Stoessel, D., Sagerer, G., 2006. Kernel particle filter for visual quality inspection from monocular intensity. In: Franke, K., Muller, K.R., Nickolay, B., Schafer, R.
(Eds.), Proceedings of Pattern Recognition: Lecture notes in computer science. Springer-Verlag, Berlin, pp. 597-606. 4174.

Tartakovsky, A., Meakin, P., 2005. A smoothed particle hydrodynamics model for miscible flow in three-dimensional fractures and two-dimensional Rayleigh-Taylor instability. J. Comput. Phys. 207, 610-624.

Wand, M.P. Jones, M.C. 1993. Comparison of smoothing parametrizations in bivariate kernel density estimation. J. Am. Stat. Assoc. 88, 520-528.

Wand, M.P., Jones, M.C., 1994. Multivariate plug-in bandwidth selection. Comput Statist 9, 97-116.

Willmann, M., Carrera, J., Sanchez-Vila, X., 2008. Transport upscaling in heterogeneous aquifers: what physical parameters control memory functions? Water Resour. Res. 44, W12437. doi:10.1029/2007WR006531.

Willmann, M., J. Carrera, X. Sanchez-Vila, O. Silva (2009), Coupling of mass transfer and reactive transport for non-linear reactions in heterogeneous media,, Water Resour. Res., In Press. 